\title{
The Effects of Conjugated Linoleic Acid and Berberine Supplementation on Markers of Allopurinol Activated Oxidative Stress in Broiler Chickens
}

Vincent Dartigue

Follow this and additional works at: https://researchrepository.wvu.edu/etd

\section{Recommended Citation}

Dartigue, Vincent, "The Effects of Conjugated Linoleic Acid and Berberine Supplementation on Markers of Allopurinol Activated Oxidative Stress in Broiler Chickens" (2017). Graduate Theses, Dissertations, and Problem Reports. 5436.

https://researchrepository.wvu.edu/etd/5436

This Thesis is protected by copyright and/or related rights. It has been brought to you by the The Research Repository @ WVU with permission from the rights-holder(s). You are free to use this Thesis in any way that is permitted by the copyright and related rights legislation that applies to your use. For other uses you must obtain permission from the rights-holder(s) directly, unless additional rights are indicated by a Creative Commons license in the record and/ or on the work itself. This Thesis has been accepted for inclusion in WVU Graduate Theses, Dissertations, and Problem Reports collection by an authorized administrator of The Research Repository @ WVU. For more information, please contact researchrepository@mail.wvu.edu. 


\title{
The effects of conjugated linoleic acid and berberine supplementation on markers of allopurinol activated oxidative stress in broiler chickens
}

\author{
Vincent Dartigue \\ Thesis submitted to the \\ Davis College of Agriculture, Natural Resources and Design \\ At West Virginia University
}

in partial fulfillment of the requirements for the degree of

Master of Science in

Animal and Food Sciences

Hillar Klandorf, PhD., Chair

Kimberly Barnes, PhD

Knox VanDyke, PhD

Division of Animal and Nutritional Sciences

Morgantown, WV

2017

Key words: allopurinol, oxidative stress conjugated linoleic acid, berberine, inflammation, antioxidants

Copyright 2017 Vincent Dartigue 


\title{
ABSTRACT \\ The effects of conjugated linoleic acid and berberine supplementation on markers of oxidant status in broiler chickens
}

\author{
Vincent Dartigue
}

\begin{abstract}
Selection for rapid growth in poultry can be linked to an exaggerated state of oxidative stress (reactive oxidative species, ROS). Reactive oxidative species are kept in balance by endogenous and exogenous antioxidants. Two compounds, conjugated linoleic acid (CLA) and berberine (BRB) a purified compound from plant root extract have been suggested to ameliorate antioxidant activity. A ten week study examined the effect of CLA and berberine supplementation on markers of oxidative stress in poultry. Broiler chickens $(n=60)$ were equally divided into six groups; a control, a CLA group where half of the regular oil used in a standard was substituted for a CLA oil mixture of cis-9 trans-11 and trans-10 cis-12 isomers, a berberine group (BRB) consisting of berberine supplementation $(200 \mathrm{mg} / \mathrm{Kg}$ feed), an allopurinol (ALLO) group $(25 \mathrm{mg} / \mathrm{kg}$ body weight), a CLA and allopurinol (CLA+ALLO) with the same dose as the CLA and allopurinol groups and a berberine and allopurinol group (BRB+ALLO) ( $200 \mathrm{mg} / \mathrm{Kg}$ feed and $25 \mathrm{mg} / \mathrm{kg}$ body weight, respectively). The allopurinol was added to help induced an oxidative stress state. Body weight, plasma uric acid, plasma glucose, and relative gene expression of six endogenous liver antioxidants were measured during the course of the study. Neither berberine nor CLA had an effect on blood glucose concentration. The addition of allopurinol (ALLO) to the diet induced an oxidative stress state as measured by a significant reduction in plasma uric acid. There was a 10-fold increase in the relative mRNA expression of superoxide dismutase 2 and 3 as well as glutathione 1 and 3 in CLA+ALLO and BRB+ALLO treatment groups. Notably, CLA increased the expression of uncoupling protein 34-fold compared to control while the addition of allopurinol blocked these changes. In contrast, there was a slight increase in the BRB+ALLO treatment. No significant changes were measured in blood glucose. In the case of plasma ceramides, there was significant decrease in concentration in the redox groups compared to controls. Despite the increase in mRNA expression of the antioxidants genes, these results suggest that at the dosages administered, CLA and berberine were not effective in reversing the oxidative stress induced by allopurinol. Despite the increase in mRNA expression of the antioxidants genes, these results suggest that at the dosages administered, CLA and berberine were not effective in reversing the oxidative stress induced by allopurinol.
\end{abstract}




\section{Acknowledgements}

First, I would like to thank Dr. Klandorf for giving me this opportunity, for his guidance and his unwavering support throughout my graduate studies at West Virginia University. Second, I would like to thank Dr. Barnes for giving me the chance as an undergrad to work in her lab, which where I first got a glimpse of research. And also thank you to Dr. VanDyke, committee member, for his insight in research and the use of his lab.

I would like to thank Elizabeth Falkenstein for her help in the lab and at the farm. The Stewartown farm crew, Fred Roe and Rick Wood. I would also like to thank Dr. Yao and Pransanthi Koganti for their help with the PCR analysis, Dr. McFadden, Dr. Rico and Zach Phipps for their help and insight into the ceramides analysis.

Last but not least, my family for their complete support. West Virginia is not around the corner, but they were willing to support my decision, they encourage me to push forward and also provided a group to bounce back ideas over the phone, always telling me to study and prepare the topic I least wanted on the exams: paid off. Big thanks to my sister, Genevieve who was willing to review some of my biology essays in the middle of the week. 


\section{Table of Contents}

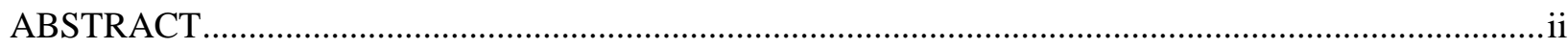

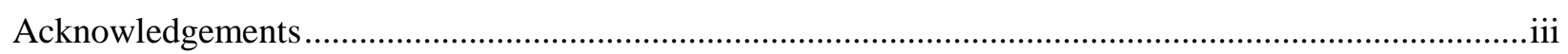

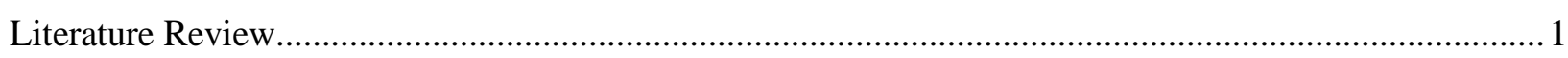

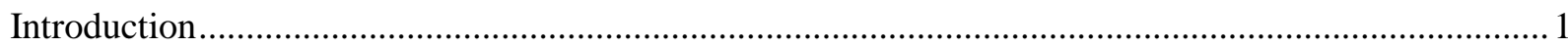

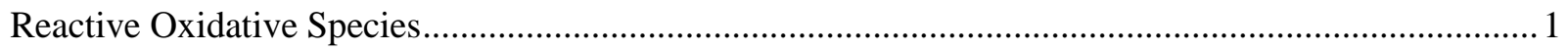

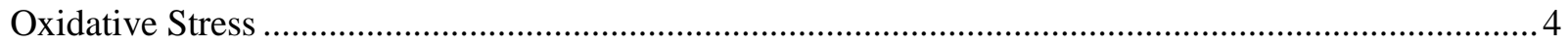

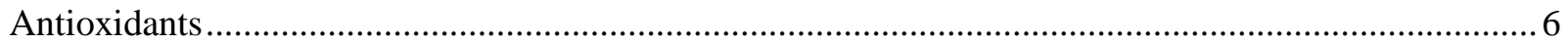

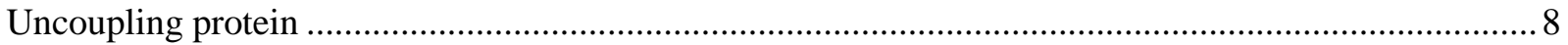

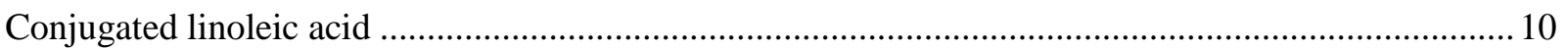

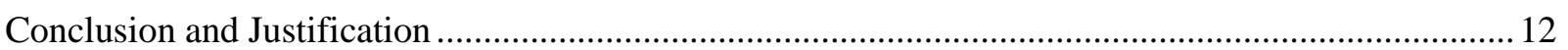

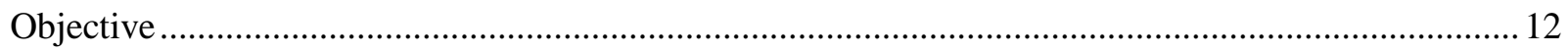

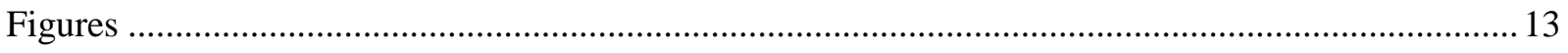

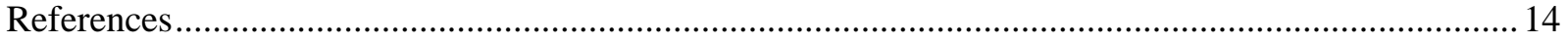

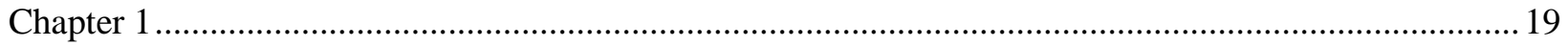

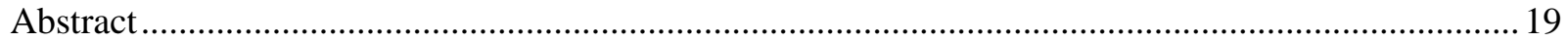

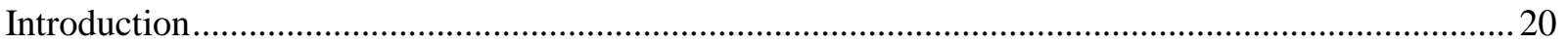

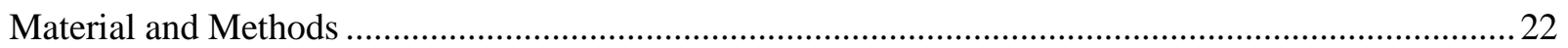

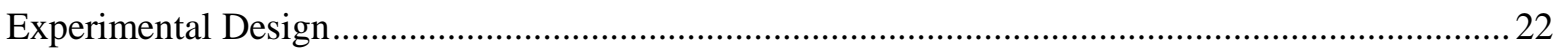

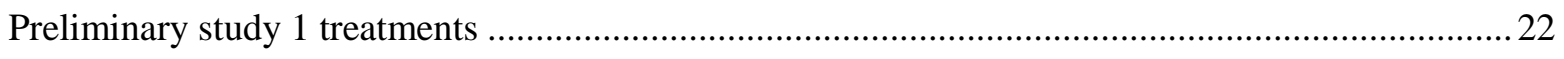

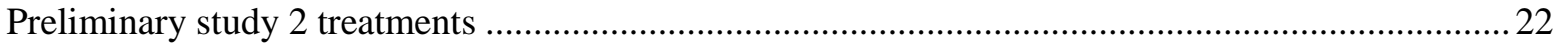

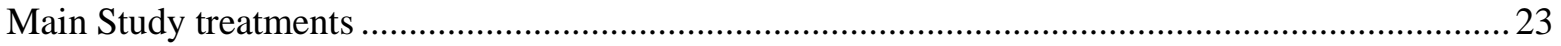

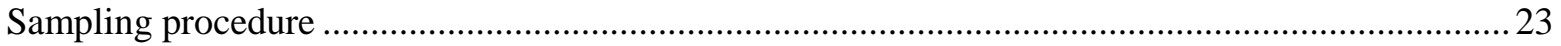

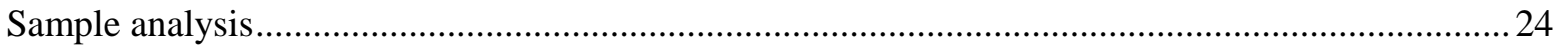

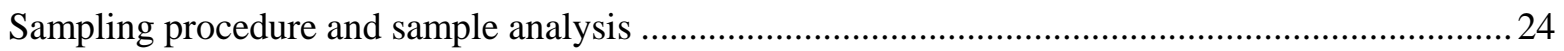

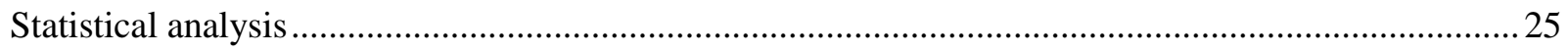

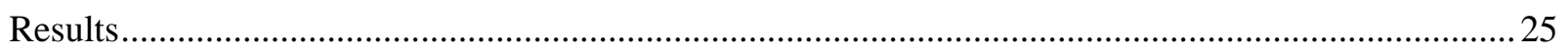

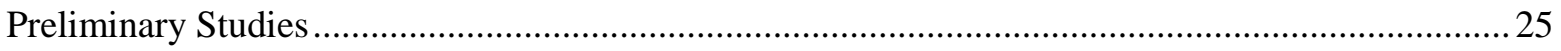

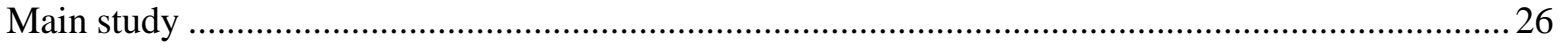

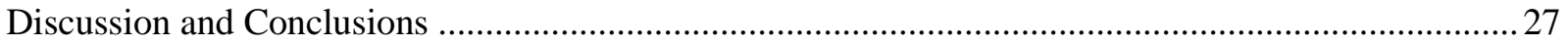

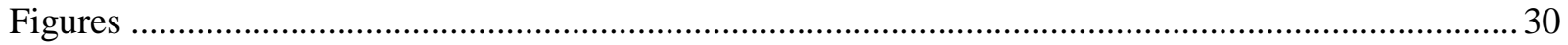




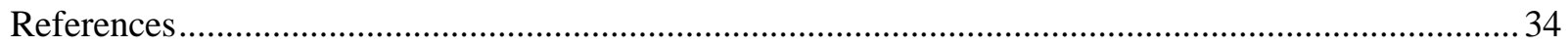

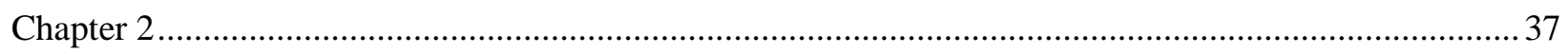

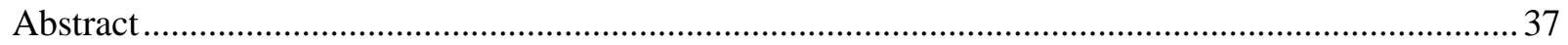

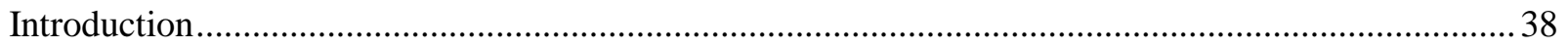

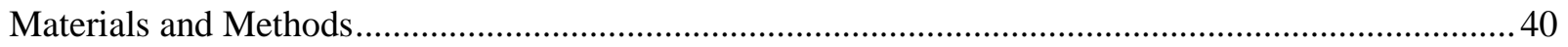

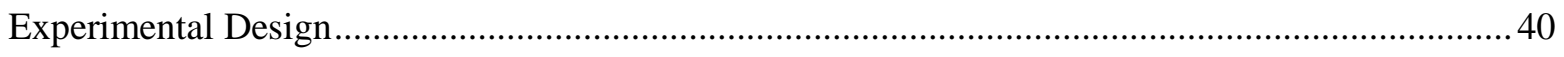

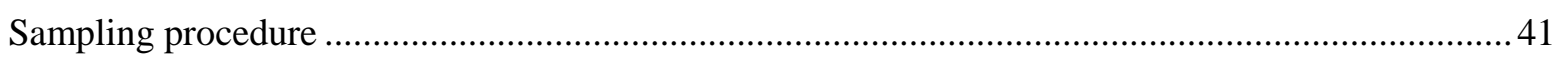

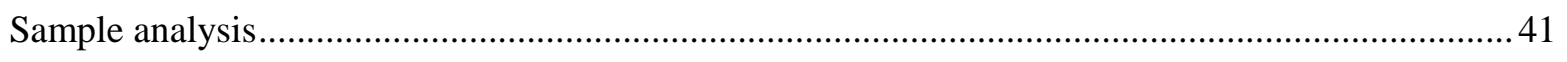

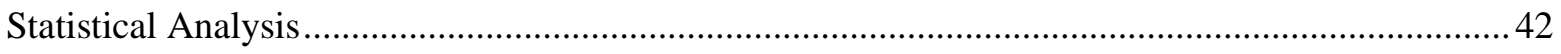

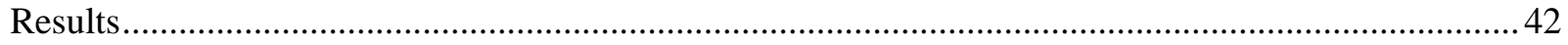

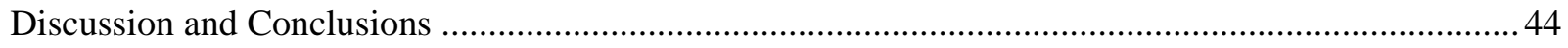

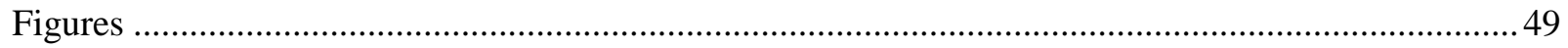

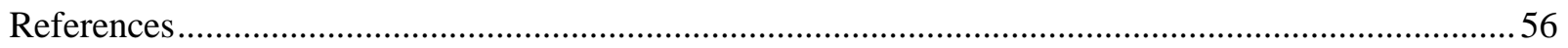




\section{List of Tables}

Chapeter ONE

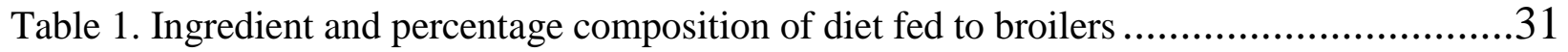

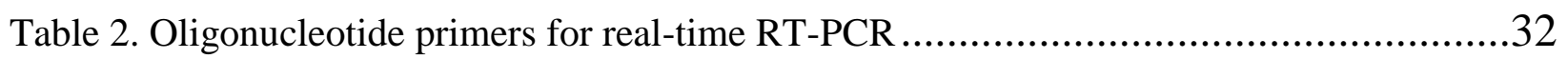

Table 3. Plasma glucose at the beginning and last week of sampling ..............................32 
Literature Review

\section{List of Figures}

Figure 1. Uric acid keto-enol tautomerization ..................................................................... 13

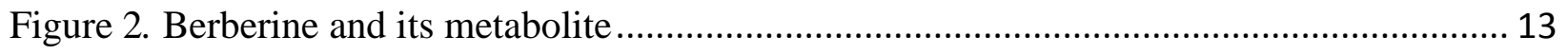

Figure 3. Linoleic and conjugated linoleic acid .......................................................... 13

Chapter ONE

Figure 1. Change in plasma uric acid over the course of the last four weeks of treatments......... 33

Figure 2. Relative mRNA expression of markers of oxidative stress in liver of chickens .......... 33

Chapter TWO

Figure 1. Changes in plasma ceramides, PUA, NEFA and weight in control birds over time ..... 52

Figure 2. Treatment effects on body weight, plasma ceramides, PUA and NEFA .................... 55 


\section{Literature Review}

\section{Introduction}

Inflammation is a vital and crucial part of physiology and the immune system. It is characterized physiologically by redness, heat, soreness and rashes. At the molecular level, it is broader: from diapedesis to the chemical reactions and signaling responses involved in the process. The term inflammation can be further divided into an acute or chronic response. Acute inflammation can be defined as a short and pronounced response whereas it is sustained in the chronic response; which can be deleterious. Chronic inflammation has been linked to metabolic syndrome. And the term metabolic syndrome refers to a set of components such as hyperglycemia, hypertension and hypertriglyceridemia. In a high fat diet, excessive circulating fatty acids are not taken up by adipose tissue. These circulating fatty acids have signaling capabilities which have been shown to activate proinflammatory factors as part of the innate immune system which result in a state of inflammation. This component of the immune response utilizes reactive oxidative species to destroy foreign or dysfunctional molecules and place the subject in a control and temporary state f oxidative stress: the production of reactive oxidative species is increased, controlled and used toward a specific end (Esposito et al., 2004, Sutherland et al., 2004, Serhan et al., 2005, Monteiro et al., 2010).

\section{Reactive Oxidative Species}

Reactive oxidative species (ROS) are highly reactive oxygen based molecules. They include molecules such as superoxide anion $\left(\mathrm{O}_{2}^{-}\right)$, hydroxyl radical $\left(\mathrm{OH}^{-}\right)$, hydrogen peroxide $\left(\mathrm{H}_{2} \mathrm{O}_{2}\right)$, nitric oxide (NO), peroxynitrite $\left(\mathrm{ONOO}^{-}\right)$and singlet oxygen $\left({ }^{1} \mathrm{O}_{2}\right)$ (Carocho et al. 2013).

From a metabolic point of view, ROS are generated by endogenous and exogenous pathways. Endogenous ROS are produced by electron leakage from the mitochondria or other organelles such as peroxisomes, and chloroplast in plants (Apel et al. 2004). Mitochondrial production of ROS starts as an 
electron leak from the NADH dehydrogenase complex also known as Complex I and the CoQ reductase complex, complex III of the electron transport chain (ETC), the latter having the highest rate of production as demonstrated by Qun et al. (2003). If there is a build-up of substrate in Complex I, the rate of electron transfer from ubiquinone to cytochrome $\mathrm{C}(\mathrm{Cyt} \mathrm{C})$ in Complex III is reduced as Cyt $\mathrm{C}$ can only carry one electron at a time (Mujahid et al. 2009). As the build-up of substrate continues in Complex I, more electrons are presented to Complex III. Under normal conditions, the semiquinone would be re-reduced to ubiquinol by reacting with a proton present in the matrix. However, in the situation where there is an electron leak (before semiquinone can be reduced), the electron reacts with an oxygen molecule also present in the matrix creating a superoxide anion. (Albert et al. 2002)

Another source of endogenous ROS production is through a process called "oxidative burst." This process is mostly used in host immunity. The oxidative burst involves the generation of hydrogen peroxide and superoxide by nicotinamide adenine dinucleotide phosphate-oxidase enzyme (NADPH-oxidase, NOX). NOX is a family of transmembrane proteins involved in the reduction of oxygen to superoxide anion or hydrogen peroxide based on the homologue or type of activation triggered. The NOX family can be activated by several factors but its reduction capacity is limited by the amount of available NADPH, the reducing agent as well as oxygen, the oxidizing agent. (Apel et al. 2004). The production of hydrogen peroxide is thought to occur mostly when NOX is constitutively activated. Hydrogen peroxide, if left unchecked, can split and form hydroxyl radical.

Exogenous ROS can also be produced by factors such as radiation and environmental factors such as chemical oxidants, hyperoxia toxins and chemotherapeutics (Bouzid et al. 2015). Once such chemotherapeutic is allopurinol. Allopurinol is a purine based analogue of hypoxanthine; it is a potent inhibitor of xanthine oxidase and thus is used primarily to treat gout in humans. It does so by inhibiting plasma uric acid (PUA) production, which itself is an antioxidant (Klandorf et al. 1999). It has been suggested that the long life span of birds compared to other animals of similar size can be associated to the concentration of circulating UA, which limits tissue damage (Holmes et al. 2001). 
Reduction and oxidation (Redox) reactions are one of the major chemical pathway by which regulation of biological pathways occurs in cells (Ray et al. 2012). Although ROS generation is considered to be a byproduct of respiration it is highly regulated and kept in control by antioxidants. Because of the extensive regulation of ROS production it is not surprising that they can also be used in signaling pathways as second messengers involved in intracellular signaling (Martin et al. 2002, Foo et al. 2011). ROS messengers have unusual attributes and properties in the way they go about signaling. In conventional signaling, the substrate binds its receptor in a non-covalent way, which leads to a response. Reactive oxidative species tend to create a covalent bond with their target resulting in a modification/structural alteration in the receptor. Situations where ROS are used as a second messenger, the target is expected to be modified with no unwanted and unforeseen impact/activity. After the oxidized receptor has generated its response, it's degraded in order to prevent collateral damage. However, in the case of unplanned protein, lipid or DNA oxidation, there is no immediate regulation of these oxidized molecules, thus the structural alteration may lead to an undesired activity or cell response. For example, the alteration can lead to a change in cell state, leading to a non-homeostatic cell, which can be characterized by the overexpression of oncogenes, formation of mutagens and overall increase in inflammation (Martin et al. 2002, Wu et al. 2006, D’Autréaux et al. 2007, Pisoschi et al. 2015). In the case of an electron leak (and not as second messengers) ROS do not have specific targets, however, they exhibit an affinity for some types of molecules/patterns. For example, superoxide anion $\left(\mathrm{O}_{2}^{-}\right)$has a high affinity for iron-sulfur clusters $(\mathrm{Fe}-\mathrm{S}) ; \mathrm{O}_{2}^{-}$oxidizes the clusters at a high rate thus preventing their diffusion. All the $\mathrm{O}_{2}{ }^{-}$anions either oxidize a Fe-S cluster, or dismute to hydrogen peroxide, factors that limit its signaling capabilities. Hydrogen peroxide is much more versatile, it is uncharged compared to $\mathrm{O}_{2}^{-}$, which allows it to diffuse across membranes and thus can activate a variety of second messengers (D’Autréaux et al. 2007, Veal et al. 2007). Further, hydrogen peroxide can elicit different responses dependent on its concentration. For example, Veal et al. (2007) suggested that in Schizosaccharomyces pombe different levels of $\mathrm{H}_{2} \mathrm{O}_{2}$ activate separate transcription responses. In another case, Sablina et al. (2005) reported that low levels of hydrogen peroxide triggers an antioxidant response whereas high levels induces apoptosis of the cell by increased expression of pro-oxidants. These limited 
findings suggest that more research is needed on ROS signaling. However, in the botanical sciences it is widely accepted that ROS such as hydrogen peroxide and nitric oxide are key molecules involved in signaling pathways, both in biotic and abiotic factors but with a tight-regulation of antioxidants that limit the shift towards oxidative stress (Neil et al. 2001).

\section{Oxidative Stress}

Oxidative stress arises when the balance between oxidants (ROS) and antioxidants favors the formation of oxidants (Reuter et al. 2010, Settle et al. 2014). This imbalance can be characterized by a chronic/sustained state of inflammation. At the molecular level, oxidative stress causes DNA damage, lipid peroxidation and protein damage. The DNA, lipid or protein loses or gains an unwanted covalent bond formed by reaction with a ROS. High concentrations of damaged molecules can contribute to the inflammatory process by directing the cell away from the homeostatic state as seen in neurodegenerative diseases (Uttara et al., 2009). Another mechanism by which oxidative stress is linked to inflammation is the build-up of ROS, which activates some transcription factors that in turn leads to the production of inflammatory cytokines and chemokines. The localized presence of elevated levels of inflammatory cytokines and chemokines tend to attract macrophages that attack the host cells. Over a period of time, these factors contribute to chronic disease complications associated with cancer, diabetes or atherosclerosis for example (Reuter et al 2010). Thus, the concentration of ROS increases in the pancreatic beta cells there is an increase in macrophage infiltration that overtime causes the destruction of the beta cells effectively ceasing the production of insulin (Giongo et al. 2011, Sun et al. 2015).

An increase in ROS production is usually the cause of inflammation, which if chronic is causes the disease itself such as inflammatory bowel disease or even aging. With this predetermined cascade of events; identifying oxidative stress markers resulting from ROS mediated oxidation represents a useful means of diagnosis. One classic method is the measurement of lipid peroxidation via thiobarbituric acid reactive substances, mainly malondialdehyde, which results from the decomposition of lipid peroxide 
(Papastergiadis et al. 2012). With the development and increased use of mass spectrometry, the greater sensitivity of the technique has permitted the detection of useful biomarkers.

Ceramides and isoprostanes are 2 categories of lipids that have been linked with oxidative stress (Bikman et al. 2011). Ceramides are sphingolipids, composed of a sphingosine back-bone attached to a fatty acid. They are readily present in blood and in every tissue, being a major component of the cell wall. Their production occurs via 3 major pathways: de novo synthesis, sphingomyelin hydrolysis and the salvage pathway. The de novo synthesis pathway involves the addition of a serine amino acid to fatty acyl CoA by the enzyme serine palmitoyltransferase (SPT). The product of this reaction undergoes three further reactions before being an active ceramide. The other two pathway of ceramide production are through ceramide recycling. Sphingomyelin hydrolysis is a pathway in which the phosphorylcholine group is hydrolyzed from the sphingomyelin by sphigomyelinase (SMase). The third means of producing ceramide is through the salvage pathway where sphingolipids are degraded in lysosome by acidic SMase to generate ceramides.

An increase in circulating ceramides concentration has been positively correlated to stress stimuli and ROS generation, the primary path of production of ceramides being de novo synthesis, the second being the salvage pathway (Bikman et al. 2011). Other studies have shown that an increase in circulating ceramide concentration is associated with decrease insulin sensitivity, thus a decrease in nutrient uptake by the cell (Evan et al. 2002, Rico et al. 2016). Nutrient uptake and insulin insensitivity are linked by the ability of ceramide to down-regulate Protein kinase B/Akt. Ceramide can inhibit Akt, a serine threonine protein kinase, an enzyme that is involved in cell metabolism through the activation of protein phosphatase $2 \mathrm{~A}$ (PP2A) which dephosphorylates Akt. Alternatively Akt activity is decreased by ceramides via Protein kinase $\mathrm{C}$ zeta intermediary (PKC $\zeta$ ) (Andrieu-Abadie et al. 2001, Summers et al. 2006, Bikman et al. 2011).

Isoprostanes are endogenous prostaglandin-like (PG) compounds derived from arachidonic acid as a result of peroxidation with ROS independently of the cyclooxygenase enzyme pathway (COX) (Morrow et al. 1992, Liu et al. 2009). Isoprostanes are mainly isomers of prostaglandins $\mathrm{F}_{2 \alpha}\left(\mathrm{PGF}_{2 \alpha}\right)$ that are biologically 
active (Morrow et al 1992, Montuschi et al. 2004). Their measurement is a reliable indicator of oxidative stress. An increase in isoprostane concentrations is positively correlated with oxidative injury and is not affected by dietary lipids (Montuschi et al. 2004). Isoprostanes are equally present in both tissue and fluids and their presence in both tissues and fluid makes for a reliable indicator of oxidative stress (Bessard et al. 2001, Montuschi et al. 2004).

Oxidative stress, as previously discussed, can be initiated by a variety of factors including a positive energy balance or heat stress, which includes an increase in body temperature and/or poor nutrient uptake which can be linked to an increase in metabolic activity. Birds are known to naturally have a higher rate of metabolism, body temperature, blood glucose, and double folded mitochondria, all of which would suggest an increase in production of ROS compared to mammals. However, birds tend to live longer than mammals of the same relative body size due to the production of various endogenous antioxidants including uric acid (Carro et al. 2010).

\section{Antioxidants}

Antioxidants (AOx) consist of a diverse range of molecules that limit the oxidation of substrates (Carocho et al. 2013, Oyewole et al. 2015). From proteins such as superoxide dismutase to simpler organic compounds, such as uric acid, they are all viewed as scavenger molecules with the ability to form a stable compound after being oxidized. In normal conditions, AOx are there to maintain balance thus limiting the shift to oxidative stress status. Just like the oxidants, AOx can be classified as endogenous and exogenous (Settle et al. 2015, Pisoschi et al. 2015).

Endogenous antioxidants are scavenging molecules/enzymes and include among them superoxide dismutase (SOD), catalase, glutathione peroxidase (GPX) and uric acid. Their primary purpose is to reduce ROS to a non-toxic state by functioning alone or working in concert (Larson et al. 1988). Enzymes such SOD have a metal base as a cofactor, in which is capable of accepting an electron. In the case of SOD, the metal bases are present in function of the location where SOD is synthesized; for example, iron (Fe), 
cupper/zinc $(\mathrm{Cu} / \mathrm{Zn})$ bases are largely found in the cytosol whereas the manganese $(\mathrm{Mn})$ base is mostly found within mitochondria (Blokhina et al. 2003). SOD accelerates the dismutation of superoxide radicals to the lesser toxic form, hydrogen peroxide, which is then converted to water and $\mathrm{O}_{2}$ by catalase (Halliwell et al. 1996, Blokhina et al. 2003).

Another way to reduce oxidants is to donate the electrons in the form of protons. As an endogenous scavenger, glutathione peroxidase (GPX) works in this fashion. The reaction usually involves two glutathiones: the thiol group from each glutathione donates the proton which results in the formation of a disulfide bridge, glutathione sulfide (GSSG). Glutathione sulfide is then recycled/reduced by NADPH via glutathione reductase (Fang et al. 2002).

Plasma uric acid (UA) although not a protein is considered a potent antioxidant. UA is a product of purine degradation by way of xanthine oxidase metabolism of hypoxanthine and xanthine (Carro et al 2010, Fabbrini et al 2014). Its antioxidant properties are associated with the enol form of uric acid. After undergoing tautomerization, hydroxyl groups are produced which then react in the same mode of action as glutathione by donating protons (Figure. 1). The synthesis of uric acid can be reliably blocked via allopurinol administration, which offers a means to increase oxidative stress in broiler chickens (Klandorf et al 2001).

Exogenous antioxidants on the other hand are non-protein based and are mostly found in fruits and vegetables. Pishoschi et al (2015) suggested that exogenous antioxidant can be divided into four major classes: two of the four are comprised of vitamin $\mathrm{C}$ and $\mathrm{E}$ and their variants, while the othe two are carotenoids and phenolic based compounds that include several subgroups. In addition to these four major classes other compounds like minerals (selenium), and alkaloids compounds such as berberine may exert antioxidant properties (Baraboi et al., 1999, Li et al 2014).

Berberine (Figure. 2) is derived from Coptidis Rhizoma and Phellodendri cortex (Kuo et al 2004). This compound is primarily used as an antidiarrheal in traditional Chinese medicine (Xu et al 2014). In 
addition, it possesses several known pharmaceutical properties which range from anti-cancer effects, to anti-diabetic and anti-inflammatory effects. It is not only considered to have direct antioxidant properties but can also elicit an increase in endogenous antioxidant production (Li et al 2014, Liu et al 2015, Xie et al 2015). Berberine has been suggested to increase the activity of AMP activated protein kinase (AMPK) pathway and to decrease the mitogen activated protein kinase (MAPK) pathway as well as inhibit cyclooxygenase-2 and the necrosis factor- $\kappa \mathrm{B}(\mathrm{NF}-\kappa \mathrm{B})$ pathway (Kuo et al. 2003, Lee et al. 2006, Li et al. 2014, Fan et al. 2015).

Several factors have been suggested to limit the effectiveness of Berberine, among them is it rate of absorption. Berberine is not a readily soluble compound, in fact its solubility and thus its absorption is inversely related to its purity. Ma et al. (2016) suggested that Coptidis Rhizoma extract is more soluble and thus readily absorbed when compared to pure berberine. However, the extract is also accompanied by nanoparticles that offset its effectiveness.

ROS neutralization by antioxidants, although effective, is not the only approach to keep them in balance. Another mechanism to target them is to decrease the initial production of ROS. Hence, reducing the electron leak in the ETC, the site of production in the mitochondria by uncoupling protein (UCP), represents an alternative approach.

\section{Uncoupling protein}

Uncoupling protein (UCP) is a transmembrane protein transporter located in the inner membrane of the mitochondria (Abe et al. 2006). UCP is a member of a family of several homologues, which include UCP1, UCP2 and UCP3 in humans and are involved in thermogenesis and/or ROS balance by moving protons across the inner mitochondrial membrane, down their concentration gradient by fatty acid flippase activity. UCP are activated by free fatty acids (FFA) and inhibited by purine nucleotides in an allosteric manner (Echtay et al. 2001, Teulier et al. 2001, Diano et al. 2012, Berardi et al. 2014). Nucleotide binding occurs at the hydrophilic region with the phosphate group near the proline-kinks. The binding sites for FFA 
are located on the outside of the protein, specifically in helix 1 and helix 6 of the transmembrane region. Fatty acyl chains are positioned in the hydrophobic region of helix 1 and 6 whereas the carboxylate head is located near the charged region close to the surface of the lipid bilayer. FFA binding to UCP results in a chemical change in the active site. Protons $\left(\mathrm{H}^{+}\right)$within the inter membrane space are translocated to the inner matrix in a FA-dependent manner with the carboxyl head of the fatty acid used as a proton acceptor.

UCP effectively uncouples respiration (ETC) from oxidative phosphorylation. It selectively decreases the gradient of protons required by complex $\mathrm{V}$ to drive ADP phosphorylation in addition to increasing the proton gradient in the mitochondrial matrix which decreases the probability of the electron on semiquinone from reacting with $\mathrm{O}_{2}$ and creating a superoxide anion (Criscuolo et al. 2005). Electrons are transported as a proton via NADH to complex I and III at which point they reduce ubiquinone (Q) to ubiquinol $\left(\mathrm{QH}_{2}\right)$. Ubiquinol cycles through complex III which presents the electron to cytochrome C (Cyt C) effectively reducing it. Cyt $\mathrm{C}$, however, can only transport one electron at a time, so ubiquinol is partially reduced to semiquinone. At this point the semiquinone, which carries the electron has two possible paths. One possibility is that the electron is "leaked" and reacts with $\mathrm{O}_{2}$, which functions as an electron acceptor, leading to a superoxide anion thus creating reactive oxidative species. A second possibility is that before the electron is leaked; semiquinone is re-reduced to ubiquinol by reacting with a proton present in the mitochondrial matrix that was moved from the inter membrane space of the mitochondria to the matrix by UCP. The ubiquinol can then, undergo a second cycle and present the second electron to Cyt $\mathrm{C}$ and proceed through the ETC (Genova et al. 2014, Baniulis et al. 2016).

UCP1 is mostly found in brown adipose tissue (BAT) with significant role in thermogenesis. A proton leak in this homologue has been associated with the generation of heat by uncoupling oxidative phosphorylation from the mitochondrial ETC (Krauss et al. 2005). This process allows energy to be released as heat. The UCP 2 and UCP3 homologues have been suggested to be involved in a reduction in ROS by the previously described mechanism. UCP 2 is expressed in several tissues whereas UCP 3 is limited to skeletal muscle and BAT and WAT (Shabalina et al 2013). 
Overall regulation of the UCP homologues has been linked to the sympathetic nervous system. In situations where there is a need for an increase in uncoupling activity, $\beta$-adrenergic receptors are activated by catecholamines. This activation leads to an increase in the uptake of fuels by the cells expressing UCP. The increase in fuels (primarily glucose) leads to an increase production of $\mathrm{NADH}$ and $\mathrm{FADH}_{2}$, which is used to increase the UCP activity.

The thermogenic capacity of UCP makes it a potential target when investigating possible factors in the treatment of obesity. Different methodologies to treat obesity include the approach of using UCP's thermogenic properties. However, obesity can be accompanied by diabetes, which is the absence or inefficient action of insulin. Herein lies the double edge source of UCP. Krauss et al. (2005) suggested that UCP can exacerbate insulin production based on the ratio ADP/ATP. As glucose is oxidized, NADH and $\mathrm{FADH}_{2}$ feed into the ETC which is coupled with oxidative phosphorylation. The increase ATP production in turn leads to the production/release of insulin. However, when oxidative phosphorylation is uncoupled from the ETC by UCP, the concentration of ATP produced does not truly represent the state of the host and so the amount insulin produced is not reflective of the actual energy balance. Thus an insufficient amount of blood glucose is taken up from circulation which results in a prolonged state of insulin, hyper-secretion by the $\beta$-cells. Ultimately this situation may result in insulin being processed and presented/ treated as a self-antigen which lead to lymphocytic infiltration of the beta cells.

Avian UCP closely resembles that of the mammalian (approximately 70\%) UCP2 and UCP3 in its affinity and its activation by free fatty acids. Amongst the possible fatty acids available that activate UCP; it has been suggested that conjugated linoleic acid is a likely candidate (Abe et al., 2006, Mujahid et al., 20006).

\section{Conjugated linoleic acid}

Conjugated linoleic acid (CLA) refers to a group of fatty acids that are 18 carbon long; the term conjugate is used to describe a mixture of 28 geometric isomers of linoleic acid (Yang et al. 2015). The 
primary source of CLA is from dairy products. Dietary linoleic acid are biohydrogenated by the ruminants' rumen microbiota, which generates the various isomers. Some of the isomers may than be incorporated into the animal's fat or milk. Chemicals synthesis of CLA from safflower and sunflower oils has been commercialized. Of the 28 conjugated systems, cis- 9 trans-11 and trans-10 cis-12 have been shown to be the forms capable of exerting metabolic effects. However, selection for the two isomers that affect metabolism is difficult as a mix of conjugated product is normally obtained (Takashi et al. 2002).

Of the two isomers having a metabolic effect, trans-10 cis-12 possesses lipolytic activity; whereas the cis-9 trans-11 isomer is anabolic and anti-inflammatory. However, both isomers do possess anticarcinogenic properties. Although $c$ is- 9 trans-11 is correlated with anti-inflammatory actions; both isomes possesses some anti-inflammatory properties. It has been suggested that CLA replaces linoleic and arachidonic acid, consequently reducing the activity of COX and LOX as mediators of inflammation. The cis-9 trans-11 isomer of CLA has been suggested to diminish the expression of both interleukin 6 and $1 \beta$, as well as the tumor necrosis factor alpha (Radmark et al. 2014; Yang et al. 2015).

Additional studies have investigated CLA's interaction with peroxisome proliferator activated receptor (PPAR) and UCP. PPAR a family of transcription factors involved in anabolism that can be activated by polyunsaturated fatty acids. This family is composed of PPAR $\alpha, \beta, \gamma_{1}$ and $\gamma_{2}$. (Ramiah et al. 2014, Viladomiu et al. 2015). The PPAR family are involved in processes such as triglyceride synthesis and fatty acid uptake by down regulating genes such as lipoprotein lipase and fatty acid synthase as well as activating fatty acid oxidation. The different homologue have different properties, with PPAR $\alpha$ involved in catabolism, fatty acid oxidation, lipoprotein transport and mostly active in the fasting state. PPAR $\gamma$ on the other hand is expressed in a fed state and is characterized by lipogenesis and fatty acid transport. PPAR $\beta$ is a modulator of PPAR $\gamma$ (Kersten et al., 2000). The PPAR family also possesses anti-inflammatory properties through various pathway, for example, interaction with NF-кB. Studies by Chen et al. (2014) and Lian et al. (2015) found that supplementing PUFAs in the diet was correlated with a decrease in markers of inflammation such interleukin and cytokines but also in NF-кB. It was Hou et al. (2012) who elucidated 
the mechanism showing that the ubiquitination of NF- $\mathrm{B}$ B by PPAR, effectively tags it for degradation (Marion-Lettellier et al. 2016).

Uncoupling protein 1 in humans plays a role in fatty acid oxidation and heat production. CLA has been linked with an increase in the activity and expression of UCP (Yang et al. 2015, Baraldi et al. 2016). As it uncouples oxidative phosphorylation, the energy is dissipated as heat. Because ATP concentrations are not indicative of the energy level, more substrate is sent to the ETC in order to reinstate/restore homeostasis. One of the sources of substrate are the fat reserves; hence the reduction of body fat associated with UCP (Yang et al. 2015).

\section{Conclusion and Justification}

Selection of fast growing chicken in the poultry industry has led to the generation of broiler chickens defined by an increased metabolism, capable of generating market standards in an average of six weeks. Heat stress which has been linked to oxidative stress, defined by increased reactive oxidative species, is a significant concern as it impacts production and the overall health of the bird (Akbarian et al. 2016; Fellenberg et al. 2006). The use of allopurinol in broiler chicken production has previously been shown to mimic the heat stress and by association the oxidative stress state with the added possibility of controlling the severity of the stress (Klandorf et al. 2001, Simoyi et al. 2002, and Settle et al. 2015). With this background and knowledge, we posited that the use of compounds, such conjugated linoleic acid and berberine would decrease reactive oxidative species, and thus partially ameliorate the oxidative stress state induced by allopurinol (Dartigue et al. submitted).

\section{Objective}

To determine the effect of CLA and berberine supplementation on markers of oxidant status in broiler chickens 
Figures

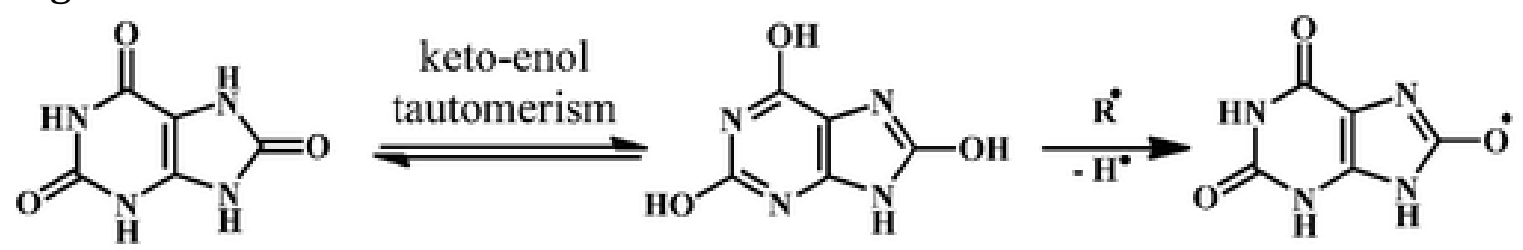

Figure 1. Uric acid keto-enol tautomerization (adapted from Nimse, 2015)

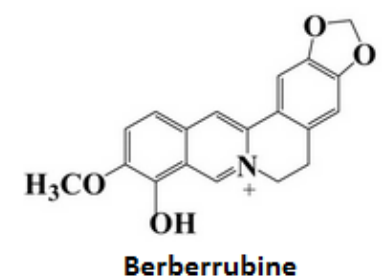

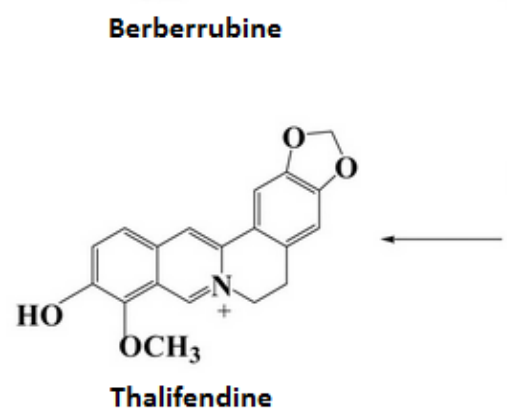

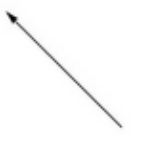<smiles>COc1ccc2c(c1OC)C[C@H]1Cc3cc4c(cc3C1=C2)OCCO4</smiles>

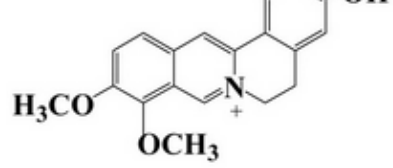

Demethyleneberberine

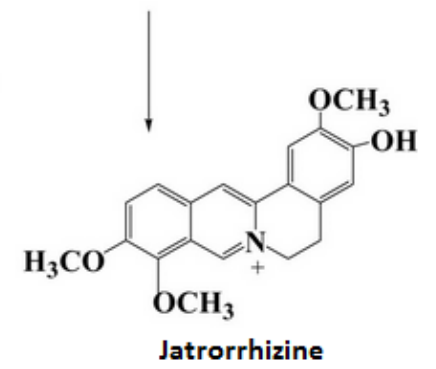

Figure 2. Berberine and its metabolite (adapted from https://examine.com/supplements/berberine/)

Linoleic Acid, 18:2 n-6

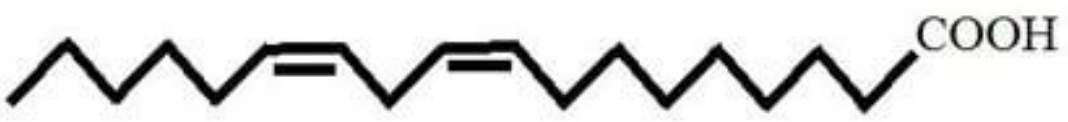

CLA, 18:2 c-9, t-11

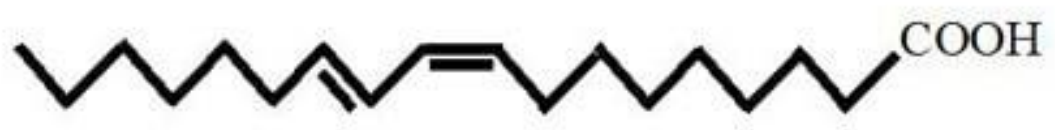

CLA, 18:2 t-10, c-12

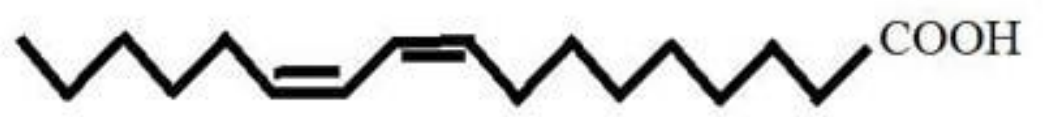

Figure 3. Linoleic and conjugated linoleic acid (https://authoritynutrition.com/conjugated-linoleic-acid/) 


\section{References}

1) Abe T, Mujahid A, Sato K, Akiba Y, Toyomizu M. Possible role of avian uncoupling protein in downregulating mitochondrial superoxide production in skeletal muscle of fasted chickens. FEBS Lett 2006 9/4;580(20):4815-4822.

2) Akbarian A, Michiels J, Degroote J, Majdeddin M, Golian A, Smet S. Association between heat stress and oxidative stress in poultry; mitochondrial dysfunction and dietary interventions with phytochemicals. Journal of Animal Science and Biotechnology 2016;7(1):37.

3) Alberts B, Johnson A, Lewis J, Raff M, Roberts K, Walter P editors. Molecular Biology of the cell. 4th ed. New York: Garland Science; 2002.

4) Andrieu-Abadie N, Gouazé V, Salvayre R, Levade T. Ceramide in apoptosis signaling: relationship with oxidative stress. Free Radical Biology and Medicine 2001;31(6):717-728.

5) Apel K, Hirt H. Reactive oxygen species: metabolism, oxidative stress, and signal transduction. Annu.Rev.Plant Biol. 2004;55:373-399.

6) Baniulis D, Hasan SS, Miliute I, Cramer WA. Mechanisms of superoxide generation and signaling in cytochrome bc complexes. Cytochrome Complexes: Evolution, Structures, Energy Transduction, and Signaling: Springer; 2016. p. 397-417.

7) Baraboĭ VA, Shestakova EN. Selenium: the biological role and antioxidant activity. Ukrains' kyi biokhimichnyi zhurnal (1999). 2003 Dec;76(1):23-32.

8) Baraldi FG, Vicentini TM, Teodoro BG, Dalalio FM, Dechandt CR, Prado IM, et al. The combination of Conjugated Linoleic Acid (CLA) and extra virgin olive oil increases mitochondrial and body metabolism and prevents CLA-associated insulin resistance and liver hypertrophy in C57Bl/6 mice. J Nutr Biochem 2016;28:147-154.

9) Berardi M, Chou J. Fatty Acid Flippase Activity of UCP2 Is Essential for Its Proton Transport in Mitochondria. Cell Metabolism 2014 9/2;20(3):541-552.

10) Bessard J, Cracowski J, Stanke-Labesque F, Bessard G. Determination of isoprostaglandin F2 $\alpha$ type III in human urine by gas chromatography-electronic impact mass spectrometry. Comparison with enzyme immunoassay. Journal of Chromatography B: Biomedical Sciences and Applications 2001 4/25;754(2):333-343.

11) Bikman BT, Summers SA. Ceramides as modulators of cellular and whole-body metabolism. J Clin Invest 2011 Nov;121(11):4222-4230.

12) Blokhnia O, Virolaine E, Fagerstedt KV. Antioxidants, Oxidative Damage and Oxygen Deprivation Stress: a Review. Annals of Botany 2003 January 02;91(2):179-194.

13) Bouzid M. Radical oxygen species, exercise and aging: an update. 2015(- 9):- 1245-1261.

14) Carocho M, Ferreira ICFR. A review on antioxidants, prooxidants and related controversy: Natural and synthetic compounds, screening and analysis methodologies and future perspectives. Food and Chemical Toxicology 2013 1;51:15-25. 
15) Carro MD, Falkenstein E, Radke WJ, Klandorf H. Effects of allopurinol on uric acid concentrations, xanthine oxidoreductase activity and oxidative stress in broiler chickens. Comparative Biochemistry and Physiology Part C: Toxicology \& Pharmacology 2010 1;151(1):12-17.

16) Chen L, Hu JY, Wang SQ. The role of antioxidants in photoprotection: A critical review. J Am Acad Dermatol 2012 11;67(5):1013-1024.

17) Chen Q, Vazquez EJ, Moghaddas S, Hoppel CL, Lesnefsky EJ. Production of Reactive Oxygen Species by Mitochondria: CENTRAL ROLE OF COMPLEX III. Journal of Biological Chemistry 2003 September 19;278(38):36027-36031.

18) Criscuolo F, Gonzalez-Barroso MdM, Maho YL, Ricquier D, Bouillaud F. Avian uncoupling protein expressed in yeast mitochondria prevents endogenous free radical damage. Proceedings of the Royal Society of London B: Biological Sciences 2005 The Royal Society;272(1565):803-810.

19) D'Autreaux B\&, Michel. ROS as signalling molecules: mechanisms that generate specificity in ROS homeostasis. Nature Reviews Molecular Cell Biology 2007;8(- 10):- 813.

20) Diano S, Horvath TL. Mitochondrial uncoupling protein 2 (UCP2) in glucose and lipid metabolism. Trends Mol Med 2012 1;18(1):52-58.

21) Echtay KS, Winkler E, Frischmuth K, Klingenberg M. Uncoupling proteins 2 and 3 are highly active H+ transporters and highly nucleotide sensitive when activated by coenzyme Q (ubiquinone). Proceedings of the National Academy of Sciences 2001 February 13;98(4):1416-1421.

22) Esposito K, Giugliano D. The metabolic syndrome and inflammation: association or causation?. Nutrition, Metabolism and Cardiovascular Diseases. 2004 Oct 1;14(5):228-32.

23) Evans JL, Goldfine ID, Maddux BA, Grodsky GM. Oxidative stress and stress-activated signaling pathways: a unifying hypothesis of type 2 diabetes. Endocr Rev 2002;23(5):599-622.

24) Fabbrini E, Serafini M, Colic Baric I, Hazen SL, Klein S. Effect of Plasma Uric Acid on Antioxidant Capacity, Oxidative Stress, and Insulin Sensitivity in Obese Subjects. Diabetes 2014 March 01;63(3):976981.

25) Fan G, Chang J, Yin Q, Wang X, Dang X. Effects of probiotics, oligosaccharides, and berberine combinationson growth performance of pigs. Turkish Journal of Veterinary and Animal Sciences 2015;39(6):637-642.

26) Fang Y, Yang S, Wu G. Free radicals, antioxidants, and nutrition. Nutrition 2002 10;18(10):872-879.

27) Fellenberg M, Speisky H. Antioxidants: their effects on broiler oxidative stress and its meat oxidative stability. Worlds Poult Sci J 2006;62(01):53-70.

28) Foo N, Lin S, Lee Y, Wu M, Wang Y. $\alpha$-Lipoic acid inhibits liver fibrosis through the attenuation of ROStriggered signaling in hepatic stellate cells activated by PDGF and TGF- $\beta$. Toxicology 2011 3/28;282(12):39-46.

29) Genova ML. Electron transport in the mitochondrial respiratory chain. The Structural Basis of Biological Energy Generation: Springer; 2014. p. 401-417.

30) Giongo A, Gano KA, Crabb DB, Mukherjee N, Novelo LL, Casella G, et al. Toward defining the autoimmune microbiome for type 1 diabetes. The ISME journal 2011;5(1):82-91.

31) Halliwell B. Antioxidants in human health and disease. Annu Rev Nutr 1996;16(1):33-50. 
32) Hargrave KM, Li C, Meyer BJ, Kachman SD, Hartzell DL, Della-Fera MA, et al. Adipose Depletion and Apoptosis Induced by Trans-10, Cis-12 Conjugated Linoleic Acid in Mice*. Obes Res 2002;10(12):12841290.

33) Hargrave KM, Meyer BJ, Li C, Azain MJ, Baile CA, Miner JL. Influence of dietary conjugated linoleic acid and fat source on body fat and apoptosis in mice*. Obes Res 2004;12(9):1435-1444.

34) Harman D. The free radical theory of aging. Antioxidants and Redox Signaling. 2003 Oct 1;5(5):557-61.

35) Holmes D, Flückiger R, Austad S. Comparative biology of aging in birds: an update. Exp Gerontol 2001;36(4):869-883.

36) Klandorf H, Probert IL, Iqbal M. In the defence against hyperglycamiea: an avian strategy. World's Poultry Science Journal. 1999 Sep 1;55(03):251-68.

37) Klandorf H, Rathore DS, Iqbal M, Shi X, Van Dyke K. Accelerated tissue aging and increased oxidative stress in broiler chickens fed allopurinol. Comparative Biochemistry and Physiology Part C: Toxicology \& Pharmacology 2001 6;129(2):93-104.

38) Krauss S, Zhang C, Lowell BB. The mitochondrial uncoupling-protein homologues. Nat Rev Mol Cell Biol 2005 print;6(3):248-261.

39) Kuo C, Chi C, Liu T. The anti-inflammatory potential of berberine in vitro and in vivo. Cancer Lett 2004 1;203(2):127-137.

40) Larson RA. The antioxidants of higher plants. Phytochemistry 1988;27(4):969-978.

41) Lee YS, Kim WS, Kim KH, Yoon MJ, Cho HJ, Shen Y, et al. Berberine, a Natural Plant Product, Activates AMP-Activated Protein Kinase With Beneficial Metabolic Effects in Diabetic and Insulin-Resistant States. Diabetes 2006 August 01;55(8):2256-2264.

42) Li Z, Geng YN, Jiang JD, Kong WJ. Antioxidant and anti-inflammatory activities of berberine in the treatment of diabetes mellitus. Evid Based Complement Alternat Med 2014;2014:289264.

43) Liu S, Lee H, Hung C, Tsai C, Li T, Tang C. Berberine attenuates CCN2-induced IL-1 $\beta$ expression and prevents cartilage degradation in a rat model of osteoarthritis. Toxicol Appl Pharmacol 2015 11/15;289(1):20-29.

44) Liu W, Morrow JD, Yin H. Quantification of F2-isoprostanes as a reliable index of oxidative stress in vivo using gas chromatography-mass spectrometry (GC-MS) method. Free Radical Biology and Medicine 2009 10/15;47(8):1101-1107.

45) Ma B, Yin C, Zhang B, Dai Y, Jia Y, Yang Y, et al. Naturally occurring proteinaceous nanoparticles in Coptidis Rhizoma extract act as concentration-dependent carriers that facilitate berberine absorption. Scientific Reports 2015 12/24;6:20110.

46) Marion-Letellier R, Savoye G, Ghosh S. Fatty acids, eicosanoids and PPAR gamma. Eur J Pharmacol 2016 8/15;785:44-49.

47) Martin KR, Barrett JC. Reactive oxygen species as double-edged swords in cellular processes: low-dose cell signaling versus high-dose toxicity. Hum Exp Toxicol 2002 02;21(2):71-75. 
48) Mollica MP, Trinchese G, Cavaliere G, De Filippo C, Cocca E, Gaita M, et al. c9,t11-Conjugated linoleic acid ameliorates steatosis by modulating mitochondrial uncoupling and Nrf2 pathway. J Lipid Res 2014 May;55(5):837-849.

49) Monteiro R, Azevedo I. Chronic inflammation in obesity and the metabolic syndrome. Mediators Inflamm 2010;2010:10.1155/2010/289645. Epub 2010 Jul 14.

50) Montuschi P, Barens PJ, Roberts LJ. Isoprostanes: markers and mediators of oxidative stress. The FASEB Journal 2004 December 01;18(15):1791-1800.

51) Morrow JD, Awad JA, Boss HJ, Blair IA, Roberts LJ,2nd. Non-cyclooxygenase-derived prostanoids (F2isoprostanes) are formed in situ on phospholipids. Proc Natl Acad Sci U S A 1992 Nov 15;89(22):10721 10725.

52) Mujahid A, Akiba Y, Toyomizu M. Acute Heat Stress Induces Oxidative Stress and Decreases Adaptation in Young White Leghorn Cockerels by Downregulation of Avian Uncoupling Protein. Poultry Science 2007 February 01;86(2):364-371.

53) Neill SJ, Desikan R, Clarke A, Hurst RD, Hancock JT. Hydrogen peroxide and nitric oxide as signalling molecules in plants. Journal of Experimental Botany 2002 May 15;53(372):1237-1247.

54) Oyewole AO, Birch-Machin MA. Mitochondria-targeted antioxidants. The FASEB Journal 2015 December 01;29(12):4766-4771.

55) Papastergiadis A, Mubiru E. - Malondialdehyde Measurement in Oxidized Foods: Evaluation of the Spectrophotometric Thiobarbituric Acid Reactive Substances (TBARS) Test in Various Foods. - J Agric Food Chem (- 38):- 9589.

56) Pisoschi AM, Pop A. The role of antioxidants in the chemistry of oxidative stress: A review. Eur J Med Chem 2015 6/5;97:55-74.

57) Ramiah SK, Meng GY, Sheau Wei T, Swee Keong Y, Ebrahimi M. Dietary Conjugated Linoleic Acid Supplementation Leads to Downregulation of PPAR Transcription in Broiler Chickens and Reduction of Adipocyte Cellularity. PPAR Res 2014;2014:137652.

58) Ray PD, Huang B, Tsuji Y. Reactive oxygen species (ROS) homeostasis and redox regulation in cellular signaling. Cell Signal 2012;24(5):981-990.

59) Reuter S, Gupta SC, Chaturvedi MM, Aggarwal BB. Oxidative stress, inflammation, and cancer: How are they linked? Free Radical Biology and Medicine 2010 12/1;49(11):1603-1616.

60) Roberts LJ, Morrow JD. Measurement of F 2-isoprostanes as an index of oxidative stress in vivo. Free Radical Biology and Medicine 2000;28(4):505-513.

61) Sablina AA, Budanov AV, Ilyinskaya GV, Agapova LS, Kravchenko JE, Chumakov PM. The antioxidant function of the p53 tumor suppressor. Nature medicine. 2005 Dec 1;11(12):1306-13.

62) Settle T. The role of uric acid as an antioxidant in selected neurodegenerative disease pathogenesis: A short review. - Brain Disorders \& Therapy 2014(- 3):-.

63) Settle T, Carro MD, Falkenstein E, Radke W, Klandorf H. The effects of allopurinol, uric acid, and inosine administration on xanthine oxidoreductase activity and uric acid concentrations in broilers. Poultry Science 2012 November 01;91(11):2895-2903. 
64) Settle T, Falkenstein E, Klandorf $H$. The effect of allopurinol administration on mitochondrial respiration and gene expression of xanthine oxidoreductase, inducible nitric oxide synthase, and inflammatory cytokines in selected tissues of broiler chickens. Poultry Science 2015 October 01;94(10):2555-2565.

65) Summers SA. Ceramides in insulin resistance and lipotoxicity. Prog Lipid Res 2006;45(1):42-72.

66) Sun J, Furio L, Mecheri R, van der Does A, Lundeberg E, Saveanu L, et al. Pancreatic $\beta$-Cells Limit Autoimmune Diabetes via an Immunoregulatory Antimicrobial Peptide Expressed under the Influence of the Gut Microbiota. Immunity 2015 8/18;43(2):304-317.

67) Sutherland JP, McKinley B, Eckel RH. The metabolic syndrome and inflammation. Metabolic syndrome and related disorders. 2004 Jun 1;2(2):82-104.

68) Takahashi Y, Kushiro M, Shinohara K, Ide T. Dietary conjugated linoleic acid reduces body fat mass and affects gene expression of proteins regulating energy metabolism in mice. Comparative Biochemistry and Physiology Part B: Biochemistry and Molecular Biology 2002;133(3):395-404.

69) Teulier L, Rouanet J, Letexier D, Romestaing C, Belouze M, Rey B, et al. Cold-acclimation-induced nonshivering thermogenesis in birds is associated with upregulation of avian UCP but not with innate uncoupling or altered ATP efficiency. J Exp Biol 2010 The Company of Biologists Ltd;213(14):2476-2482.

70) Uttara B, Singh AV, Zamboni P, Mahajan RT. Oxidative stress and neurodegenerative diseases: a review of upstream and downstream antioxidant therapeutic options. Current neuropharmacology. 2009 Mar 1;7(1):65-74.

71) Veal EA, Day AM, Morgan BA. Hydrogen Peroxide Sensing and Signaling. Mol Cell 2007 4/13;26(1):114.

72) Wu W. The signaling mechanism of ROS in tumor progression. Cancer and Metastasis Reviews 2006;25(4):- 695-705.

73) Xie J, Xu Y, Huang X, Chen Y, Fu J, Xi M, et al. Berberine-induced apoptosis in human breast cancer cells is mediated by reactive oxygen species generation and mitochondrial-related apoptotic pathway. Tumor Biol 2015;36(2):1279-1288.

74) Xu M, Xiao Y, Yin J, Hou W, Yu X, Shen L, et al. Berberine promotes glucose consumption independently of AMP-activated protein kinase activation. PLoS One 2014;9(7):e103702.

75) Yang B, Chen H, Stanton C, Ross RP, Zhang H, Chen YQ, et al. Review of the roles of conjugated linoleic acid in health and disease. Journal of Functional Foods 2015;15:314-325. 


\title{
Chapter 1
}

\begin{abstract}
Selection for rapid growth in poultry can be linked to an exaggerated state of oxidative stress (reactive oxidative species, ROS). Reactive oxidative species are kept in balance by endogenous and exogenous antioxidants. Two compounds, conjugated linoleic acid (CLA) and berberine (BRB) a purified compound from plant root extract have been suggested to ameliorate antioxidant activity. A ten week study examined the effect of CLA and berberine supplementation on markers of oxidative stress in poultry. Broiler chickens $(n=60)$ were equally divided into six groups; a control, a CLA group where half of the regular oil used in a standard was substituted for a CLA oil mixture of cis-9 trans-11 and trans-10 cis-12 isomers, a berberine group (BRB) consisting of berberine supplementation $(200 \mathrm{mg} / \mathrm{Kg}$ feed), an allopurinol (ALLO) group (25mg/kg body weight), a CLA and allopurinol (CLA+ALLO) with the same dose as the CLA and allopurinol groups and a berberine and allopurinol group (BRB+ALLO) ( 200mg/Kg feed and 25mg/kg body weight, respectively). The allopurinol was added to help induced an oxidative stress state. Body weight, plasma uric acid, plasma glucose, and relative gene expression of six endogenous liver antioxidants were measured during the course of the study. Neither berberine nor CLA had an effect on blood glucose concentration. The addition of allopurinol (ALLO) to the diet induced an oxidative stress state as measured by a significant reduction in plasma uric acid. There was a 10-fold increase in the relative mRNA expression of superoxide dismutase 2 and 3 as well as glutathione 1 and 3 in CLA+ALLO and BRB+ALLO treatment groups. Notably, CLA increased the expression of uncoupling protein 34-fold compared to control while the addition of allopurinol blocked these changes. In contrast, there was a slight increase in the BRB+ALLO treatment. No significant changes were measured in blood glucose. Despite the increase in mRNA expression of the antioxidants genes, these results suggest that at the dosages administered, CLA and berberine were not effective in reversing the oxidative stress induced by allopurinol.
\end{abstract}

Key words: allopurinol, antioxidants, broiler, ceramide, oxidative stress, 


\section{Introduction}

Inflammation is of significant concern in the poultry industry, it impacts production worldwide and the overall health of the chicken. Inflammation can arise from both environmental and internal factors, the former can be usually traced back to an increase in ambient temperature. The latter has a broader range of origin from species to gender and metabolism. Both environmental and internal factors are characterized by an increase in oxidative stress (Cerruti et al., 1993, Federico et al., 2007, Fellenber et al., 2006). Oxidative stress results from an imbalance between reactive oxidative species (ROS), which are highly reactive oxygen base molecules, and antioxidants (AOx). A major source ROS is the electron transport chain (ETC) (Chen et al., 2012, Murphy et al., 2009) and in an imbalance state they represent a danger to aerobic organisms because of their high reactivity and tendency to form covalent bonds with lipids, proteins and DNA (Martin et al., 2002, Wu et al., 2006, D’Autréaux et al., 2007). Any structural alterations in molecules can lead to a change in cell state, distancing itself from cell homoeostasis, which can be characterized by overexpression of oncogenes, formation of mutagens, and overall inflammation (Pisoschi et al., 2015).

Birds in general have higher level of plasma glucose than mammals which simulates/mimics a constant state of hyperglycemia. They, however, do not exhibit any noticeable side effects observed in mammals with similar concentrations. This is prevented by an elevated levels of plasma uric acid. Plasma uric acid is a potent Aox for circulating ROS in species lacking uricase. Previous studies in poultry have demonstrated that allopurinol supplementation reduces plasma uric acid concentrations thus establishing a controllable oxidative stress model (Simoyi et al., 2002). In a normal state, ROS generation is balanced by antioxidants and other molecules having AOx like properties, which target and neutralize the oxidative species. Antioxidants can be characterized as endogenous or exogenous. The main families of endogenous 
Aox are the superoxide dismutase and the glutathione family while the exogenous ones are mostly characterized by the presence of a phenol group (Pisoschi et al., 2015, Larson et al., 1987).

Berberine, an exogenous Aox isolated from plant root extract, has been used in traditional Chinese herbal medicine. This compound is a quaternary ammonium salt that possesses several benzene rings and methoxy functional groups, which allows the electron to circulate within the conjugated double bond system. In addition to increasing the activity of endogenous antioxidants, berberine is reputed to possess anti-diabetic, anti-inflammatory and anti-carcinogenic properties (Meeran et al., 2008, Li et al., 2014, Liu et al 2015, Xie et al., 2015). Neutralization of ROS may also be achieved by activation uncoupling protein (UCP). Uncoupling protein is a member of the family of the transmembrane proteins located in the inner membrane of the mitochondria homologues involved in thermogenesis and ROS balance. Its main mode of action is to uncouple respiration (ETC) from oxidative phosphorylation and it does so by moving protons from the inner mitochondrial membrane space into the matrix (Teulier et al., 2001; Abe et al., 2006). This action increases the probability of any pair of protons of reacting with superoxide radicals, thereby, creating metabolic water from catalase or glutathione peroxidase. Berardi et al. (2014) has suggested that UCP can be activated by the binding of fatty acid (C14 to $\mathrm{C} 18)$ to the receptor whereas purine nucleotides act as allosteric inhibitors. For example, trans-10 cis-12 conjugated linoleic acid (CLA) has been shown to affect energy balance through up-regulation of UCP activity (Abe et al., 2006, Mollica et al., 2014, Viladomiu et al., 2015, Yang et al., 2015). CLA refers to a collective of fatty acids, each 18 carbons long. The primary source of CLA is from dairy products and beef, lamb and goat fat by hydrogenation of dietary linoleic acid and stearic acid. Of the 28 conjugated isomers, the cis- 9 trans- 11 and trans- 10 cis-12 are produced in the greatest quantity and were shown to have the most prominent metabolic effects. The purpose of this study was to determine if the addition of berberine or CLA to the diet of broiler chicken could reverse the induction of an oxidative stress state induced by allopurinol. We hypothesized that the addition of the two compounds should increase the endogenous antioxidants possibly reverting the redox state induced by the help of the allopurinol. 


\section{Material and Methods}

All procedures and experiments were approved by West Virginia University's Institution Animal Care and Use Committee (ACUC \#15-0301.1).

\section{Experimental Design}

Three study were conducted, two preliminaries and the main study. The two preliminary (prelim) studies were conducted using forty five and seventy mixed sex day old Cobb/Cobb 500 broiler chicks, respectively. In the main study, sixty mixed sex day old Cobb/Cobb 500 broiler chicks were used. All chicks were obtained from Pilgrim's Pride, Moorefield, WV. Chicks were housed within pens and maintained under a twelve hour light-dark cycle with temperature set at $27^{\circ} \mathrm{C}$. For the first two days after hatch, chicks were kept in the same pen with a heating lamp. On the third day, chicks were divided in two pens until they reached one week of age, at which time they were tagged with leg bands and divided into six pens based on weight. The chicks were kept on a starter diet for the first four weeks. At week five of the study, the broiler chickens were started on different treatments (see Table 1).

\section{Preliminary study 1 treatments}

The first preliminary study lasted for a total of nine weeks was composed of 4 groups. The 4 treatments were composed of a control diet, a berberine diet (BRB) supplemented with berberine $(100 \mathrm{mg} / \mathrm{kg}$ of feed) (Li et al 2014), a conjugated linoleic (CLA) diet as explained in the main study. The last diet was a combination of berberine and CLA (BRB+CLA) which mirrored the BRB and CLA treatments. For the second preliminary study, the design registered four groups of eight chicks with replicates.

\section{Preliminary study 2 treatments}

For the second preliminary study, the design registered four groups of eight chicks with replicates and also lasted nine weeks. The second preliminary study 2 was composed of a control diet, a berberine diet (BRB) supplemented with berberine $(200 \mathrm{mg} / \mathrm{kg}$ of feed), an allopurinol diet (ALLO) given at a dose of $25 \mathrm{mg} / \mathrm{kg}$ body weight (Klandorf et al., 2001) and the last diet was a combination of berberine and allopurinol 
$(\mathrm{BRB}+\mathrm{ALLO})$ which mirrored the BRB treatments with the addition of allopurinol $(25 \mathrm{mg} / \mathrm{kg}$ of body weight) for the last week of the study. Groups four five through eight were treated with allopurinol to help induce a redox state (Settle et al., 2012). Weights were recorded weekly over the five weeks of experimental period. Berberine (prelim 1: chloride, purity $>99 \%$, prelim 2: chloride hydrate, purity $>99 \%$ ), the CLA, and the allopurinol for the respective studies were purchased as stated in the main study.

\section{Main Study treatments}

The main study lasted for ten weeks and was composed of a control group, a CLA group in which half of the regular oil used in the normal diet was substituted for a mixture of 50/50 cis-9 trans-11 and trans-10 cis-12 isomers CLA oil (1.1\% CLA oil diet) (Ramiah et al 2014). Group 3 (BRB) was supplemented with berberine (200mg/kg of feed) (Li et al 2014), group 4 (ALLO) was given allopurinol $(25 \mathrm{mg} / \mathrm{kg}$ of body weight) (Klandorf et al 2001), group 5 (CLA+ALLO) and 6 (BRB+ALLO) mirrored the CLA and BRB treatments with the addition of allopurinol $(25 \mathrm{mg} / \mathrm{kg}$ of body weight) for the last two weeks of the study. Groups four five and six were treated with allopurinol to help induce a redox state (Settle et al 2012). Weights were recorded weekly over the six week experimental period. Berberine (chloride hydrate, purity $>99 \%$ ) and Allopurinol were purchased from Sigma-Aldrich (Saint Louis, MO, USA). The CLA was purchased commercially (Vitamorph Labs, Highland, IN, USA) as $1200 \mathrm{mg}$ capsules. Food and water were provided ad libitum.

\section{Sampling procedure}

After two weeks on treatment, in all three studies blood samples $(4 \mathrm{ml})$ were obtained from six birds per pen from the brachial (wing) vein. Each sample was transferred to a heparinized tube and immediately placed on ice. The same birds were sampled for the next three bleedings: for a total of four bleedings. Once the samples were collected they were centrifuged at $2000 \mathrm{rpm}$ for 20 minutes at $4^{\circ} \mathrm{C}$ to collect plasma. They were stored at $-20^{\circ} \mathrm{C}$, pending analysis. On the last day of the study, the birds were euthanized and a sample of liver $(\sim 1 \mathrm{~g})$ was snap frozen in liquid nitrogen until stored in a $-80^{\circ} \mathrm{C}$ freezer for further analysis. 
Sample analysis

Plasma samples in the $-20^{\circ} \mathrm{C}$ were used for measurement of plasma glucose and plasma uric acid. Plasma glucose was measured using amperometric measurement of hydrogen peroxide $\left(\mathrm{H}_{2} \mathrm{O}_{2}\right)$ on a platinum electrode (YSI 2700 Biochemistry analyzer) (Hall et al., 1998, Klandorf et al., 2001). Plasma uric acid (PUA) was measured using Infinity ${ }^{\mathrm{TM}}$ Uric Acid Liquid Stable Reagent assay from Thermo Scientific (Waltham, MA, USA; Klandorf et al 2001).

Liver samples (75-100 mg) were used to study the relative mRNA expression of six antioxidant related genes in the superoxide dismutase (SOD) family, glutathione (GPX) family and uncoupling protein (UCP) family. A Trizol-Reagent (Invitrogen ${ }^{\mathrm{TM}}$, Carlsbad, CA) based method was used to extract and isolate total RNA. The isolated RNA was resuspended in nuclease free water and treated with DNase (Ambion ${ }^{\circledR}$, Thermo Scientific Waltham, MA, USA). Four microliters of DNase treated RNA were reverse transcribed to cDNA utilizing an iScript ${ }^{\mathrm{TM}}$ cDNA synthesis kit from Bio-Rad (Hercules, CA). A SYBR ${ }^{\circledR}$ Green Master Mix kit (Bio-Rad, Hercules, CA) method was used on a BIORAD CFX96 Real Time System (C1000 Touch Thermal cycler) to quantify the expression of SOD1, SOD2, SOD3, GPX1, GPX3 and avUCP using designed primers. The gene sequences were obtained from the ncbi data base (https://www.ncbi.nlm.nih.gov) and designed using the Primer3 Input (version 4.0 http://bioinfo.ut.ee/primer3-0.4.0/primer3/) (see Table 2). Results of real time PCR were normalized to the housekeeping gene GAPDH. Calculation and analysis of mRNA expression was performed using the efficiency corrected delta-delta Ct method (Barnes et al., 2012).

\section{Sampling procedure and sample analysis}

The sampling procedure again followed the main study with the exception of the addition of the resolving medium (Mono-Poly, MP biomedicals, LLC Solon, Ohio) was added, in a 1:1 ratio, to separate the leukocytes in both preliminary studies. Half of the sample were transferred to a new tube where a resolving medium (Mono-Poly, MP biomedicals, LLC Solon, Ohio) was added, in a 1:1 ratio, to separate the leukocytes. All the samples were centrifuged at $2000 \mathrm{rpm}$ for 20 minutes at $4^{\circ} \mathrm{C}$ to collect plasma and 
leukocytes. The plasma samples were stored at $-20^{\circ} \mathrm{C}$, pending analysis. The leukocytes were transferred to micro centrifuge tube and place on ice for immediate analysis. No liver samples were taken.

The analysis was the same with the exception of oxidative stress measurement. Oxidative stress was measured using L-012 (8-Amino-5-chloro-7-phenylpyrido[3,4-d]pyridazine-1,4-(2H,3H)dione Sodium Salt) base chemiluminescence techniques (Van Dyke et al 1987, Klandorf et al 2001). The leukocytes were activated using a phorbol 12-myristate 13-acetate (pma) (Sigma Saint Louis, MO, USA).

\section{Statistical analysis}

In preliminary studies, the effects of experimental treatments on bodyweight, plasma uric acid, plasma glucose and oxidative stress measurements were analyzed using ANOVA with treatment as main effect. Tukey's multiple comparison test was used for specific pair wise comparison.

In the main study, the effects of experimental treatments on bodyweight, plasma uric acid, and plasma glucose and gene expression were analyzed using ANOVA with treatment as main effect. Tukey's multiple comparison test was used for specific pair wise comparison. Statistical analysis for gene expression was done on $\Delta \mathrm{Ct}$, however, the $\Delta \Delta \mathrm{CT}$ measurement were used in the figures. The values presented are the LSM+SE. Statistical analysis was done using JMP program (SAS Institute Inc., Cary NC). Statistical significance was set at $P<0.05$.

\section{Results}

Preliminary Studies

In short, in the first preliminary study there was a significant difference between control and the $\mathrm{BRB}+\mathrm{CLA}$ group body weight, $2.45 \mathrm{~kg}$ and $1.95 \mathrm{~kg}$ respectively. In the second preliminary study, there no differences to report. End point plasma uric acid analysis revealed in the first prelim a significant decrease PUA in the BRB and BRB+CLA groups compared to controls, 0.30, 0.36 and $0.48 \mathrm{mM}$, respectively; in the second, we observed a significant decrease in all treatments compared to controls. The control had a 
PUA concentration of $0.44 \mathrm{mM}$ whereas the other 3 treatments averaged around $0.35 \mathrm{mM}$. In reactive oxidative species analysis, BRB group was significantly lower when compared to control.

\section{Main study}

There was no significant differences in body weight $(\mathrm{BW})$ gain between the treatments. For plasma uric acid analysis, there was a significant $(P<0.05)$ decrease in plasma uric acid concentrations towards the end of the study in groups administered allopurinol compared to the control (Fig 1). The decrease was more pronounced $(P<0.05)$ in the CLA+ALLO and BRB+ALLO groups, $0.17 \mathrm{mM}$ and $0.16 \mathrm{mM}$ respectively, compared to both ALLO $(0.27 \mathrm{mM})$ and controls $(0.43 \mathrm{mM})$. Neither berberine nor CLA had a measurable effect on plasma glucose concentrations (Table 2). In the BRB group, there was a significant decrease in liver/bodyweight ratio compared to controls (Fig 2). Allopurinol administration in ALLO, $\mathrm{CLA}+\mathrm{ALLO}$ and BRB+ALLO groups increased $(P<0.05)$ the liver/BW ratio as compared to the controls, Fig 2. However, CLA+ALLO and BRB+ALLO led to a numerical decrease in liver/bw ratio compared to ALLO group.

The relative expression of superoxide dismutase (SOD) 1 through 3, uncoupling protein (UCP) and glutathione (GPX) 1 and 3 are shown in Fig 3. The genes were chosen based on their location and their targets. SOD 1 and 3 uses $\mathrm{Cu} / \mathrm{Zn}$ as electron acceptors and were expressed in the outer mitochondrial membrane and in the cytoplasm, respectively. SOD2 has manganese as an electron acceptor and is located in the mitochondrial matrix. GPX 1 and 3 differed base on their location, intracellular and plasma soluble respectively. Only one homologue of UCP was studied. Control unit were expresses as arbitrary units and set as 1 . In the non-redox state, CLA led to a 4 fold increase in SOD1, whereas BRB slightly decrease SOD1 expression below controls (Fig 3, A). The ALLO group increased SOD1 expression by 3 fold, which was not significant. The redox state, however, caused by allopurinol led to a 6 and 12 fold increase $(P<$ 0.05) in both CLA+ALLO and BRB+ALLO. 
SOD2 expression across treatments was comparable to the changes observed in SOD1 (Fig 3, B). In the non-redox state, CLA increased SOD2 six fold, whereas BRB increased the expression two fold. However, SOD2 expression decreased in ALLO group in contrast to the increase observed in SOD1. CLA and BRB, when exposed to allopurinol led to an increase in expression of SOD2. CLA+ALLO also significantly increased SOD2 relative expression by 7 and BRB+ALLO by 16 fold $(P<0.05)$. In contrast to SOD1 and SOD2, SOD3 expression in CLA, BRB and ALLO groups were not significantly different compared to the controls (Fig 3, C). The redox state, however, increased expression of SOD3 in CLA+ALLO and BRB+ALLO, at 10 and 9 fold, respectively.

A marked increase in uncoupling protein (UCP3) expression occurred in response to the CLA treatment (34 fold increase) whereas BRB marginally affected UCP expression (3 fold increase) when compared to controls (Fig 3, D). UCP3 expression decreased in the ALLO group as compared to control. In contrast to CLA, CLA+ALLO only increased UCP expression 4 fold whereas there was a 10 fold increase in UCP3 expression in the BRB+ALLO group.

Glutathione1 (GPX1) expression was similar to that observed in the SOD3 group (Fig 3, E). In the non-redox state, CLA and BRB did not undergo significant changes in expression. In the redox state, ALLO did not change the expression of GPX1, whereas CLA+ALLO and BRB+ALLO treatments led to a 9 and 10 fold increase $(P<0.05)$, respectively. Glutathione 3 (GPX3) expression closely resembled that of SOD 2 in both the non-redox and redox state (Fig 3, F). There was a small increase in GPX3 expression in the CLA and BRB groups, as well as in the allopurinol treated birds. CLA+ALLO and BRB+ALLO modulation were pronounced with increase expression of 5 and 11 fold, respectively $(P<0.05)$.

\section{Discussion and Conclusions}

Plasma uric acid is a potent antioxidant in birds and its inhibition has been linked to an increase in ROS production in chicken (Settle et al., $2012 \&$ 2015). Previous studies have established that allopurinol is a potent inhibitor of plasma uric acid in chicken which leads to a redox state in the chicken (Klandorf et 
al., 2001, Simoyi et al., 2002). The degree or severity of the redox state can be varied depending on the dosage of allopurinol administered, resulting in a somewhat controllable model of oxidative stress for redox study. Making use of this model, we set out to test to determine if berberine and/or CLA could restore the bird to its pre-redox state caused by the administration of allopurinol.

One week after administration allopurinol, PUA declined in all treated groups. By week 10, the ALLO group had a level of plasma uric acid of $0.27 \mathrm{mM}$ compared to the control, $0.40 \mathrm{mM}$. However, this decline was unexpectedly more pronounced in both the CLA+ALLO and BRB+ALLO groups as they reached levels of 0.17 and $0.16 \mathrm{mM}$ respectively $(P<0.05)$. Kong et al (2004) suggest that berberine may have an inhibitory effect on xanthine oxidase; if so this would explains the negative synergistic effect on the plasma uric acid level.

CLA is known to increase the activity and expression of UCP (Takahashi et al., 2002) whereas berberine is suggested to reduce blood glucose concentration in Type 2 diabetes by increasing insulin sensitivity (Xing et al., 2011) as well as enhance the expression of endogenous antioxidants such as SOD (Li et al., 2014). UCP is a transmembrane protein with its active site exhibiting flippase activity to move protons. The accumulation of protons in the matrix increases the probability that a proton interacts with an electron as they cycle through complex III and IV. As the animal transitions from a normal to redox state, there should be an increase in antioxidants to neutralize the ROS and potentially dampen the effect of the shift. With this basic premise, the addition of these compounds was expected to increase the expression of antioxidant genes. To test this hypothesis, we measured the mRNA expression of six endogenous antioxidants genes. Berberine administration led to an increase in the relative expression of SOD 1, 2 and 3 by 13, 16 and 8 fold respectively. These compounds also improved mRNA expression of UCP, glutathione 1 and 3, by a 10 fold change in both UCP and GPX1 and an 11 fold change in GPX3. Although, CLA was hypothesized to affect UCP, it also increased the relative expression of other endogenous antioxidants, albeit less pronounced. Comparable results were reported by Cantwell et al. (1999) in human cell line exposed to cow milk containing CLA. 
Reactive oxyegen are kept in balance by antioxidants. The superoxide dismutase (SOD) family and glutathione are among the principal endogenous antioxidants. SOD catalyzes superoxide to hydrogen peroxide, which is then degraded to in water and oxygen by glutathione (Halliwell et al., 1996). Overall, for the SOD family, CLA led to a 7, 6 and 8 fold increase in SOD 1,2 and 3, respectively. Cellular glutathione's expression increased by 9 fold whereas the increase in extracellular glutathione was 5 fold. However, the addition of CLA to allopurinol did not result in further increase in UPC expression. Allopurinol is a purine analogue which may have inactivated UCP, which is down-regulated by purines. It is possible that allopurinol also masked the effect of CLA on UCP. This would explain the 3 fold increase in the redox state in contrast to the 34 fold increase in UCP expression without allopurinol. Furthermore CLA modulation of UCP expression could be an additional means to maintain ROS balance

An increase in ROS has been linked to liver inflammation due to the recruitment of inflammatory cells. ROS signaling leads to leukocytes infiltration which in turn leads to additional ROS production (Greenhalgh et al., 2015). In the current study, we measured an increase in liver size in the ALLO group as well as an increase in the liver to BW ratio as compared to controls. The addition of the BRB and CLA to the allopurinol treated birds was able to numerically decrease liver weight but not back to control levels. These results are in agreement with Hwang et al. (2002) who observed in rats that berberine decreased ROS production and the incidence of liver lesions by an increase glutathione production. The results suggest that at the dosages used in this study CLA and berberine were not able to completely restore to a non-redox state in birds administered allopurinol.

The data gathered in the preliminary studies were used to draw conclusion in parallel with our main study. The use of the allopurinol redox model in birds to study the effect of CLA and berberine on markers of oxidative stress was not conclusive. The liver to BW ratio suggested that the addition of CLA and berberine were able to partly reduce the inflammation of the liver but not to control levels. A further reduction in plasma uric acid by these two compounds to allopurinol fed birds was not anticipated, which suggest that additional ROS may have been produced. CLA and berberine were able to increase the relative 
mRNA expression of the selected endogenous antioxidant genes but without measurement of the gene product, we do not know if these changes have been translated/expressed. Finally, at the dosage of allopurinol used we were not able to completely revert the redox state. We conclude that the allopurinol model can be used to measure the ability of alternative compounds to ameliorate the induced oxidative stress.

\section{Figures}

Figure 1. Change in plasma uric acid (mM) over the course of the last four weeks of treatments. The vertical dashed line represents the time at which allopurinol was added for allopurinol supplemented treatments. The non-redox state is shown as solid lines in contrast to the redox state represented by dotted lines. The values represent mean and standard errors $(P<0.05)$

Figure 2. Relative mRNA expression of hepatic endogenous antioxidants enzyme in broiler chickens. (A, $\mathbf{B}$ and $\mathbf{C}$ ) Increase expression of superoxide dismutase (SOD) 1 through 3 in the CLA+ALLO and BRB+ALLO groups relative to control. (D) Effect of treatments on uncoupling protein (UCP3) expression. (E and F) Increase in Glutathione 1(GPX1) and GPX3 expression across treatments. Data expressed as mean and standard errors. The data was calculated using the $\delta-\delta \mathrm{Ct}$ method. GAPDH was used as a housekeeping gene to normalize. The asterisk indicate a significant difference from control $(P<0.05)$. 
Table 1. Ingredient and percentage composition of diet fed to broilers

\begin{tabular}{|c|c|c|}
\hline \multicolumn{3}{|c|}{ Item } \\
\hline Ingredient $\%$ & Control Diet & $\begin{array}{l}\text { CLA oil } \\
\text { Diet }\end{array}$ \\
\hline Soybean meal & 28.3 & 28.3 \\
\hline Corn & 66.2 & 66.2 \\
\hline Regular oil & 2.2 & 1.1 \\
\hline CLA & N/A & 1.1 \\
\hline Dicalcium phospate & 1.7 & 1.7 \\
\hline Calcium carbonate & 0.8 & 0.8 \\
\hline Salt & 0.25 & 0.25 \\
\hline Nutra blend $\mathrm{mix}^{\mathrm{a}}$ & 0.25 & 0.25 \\
\hline Methionine & 0.2 & 0.2 \\
\hline Monensin & 0.1 & 0.1 \\
\hline \multicolumn{3}{|l|}{ Total \% } \\
\hline M. Energy ${ }^{\mathrm{b}}(\mathrm{kcal} / \mathrm{kg})$ & 3130.5 & 3130.5 \\
\hline Protein \% & 19.5 & 19.5 \\
\hline Fat $\%$ & 4.9 & 4.9 \\
\hline Fiber $\%$ & 2.67 & 2.67 \\
\hline Calcium \% & 0.78 & 0.78 \\
\hline Phosphorous \% (total) & 0.67 & 0.67 \\
\hline $\begin{array}{l}\text { Phosphorous \% } \\
\text { (avalaible) }\end{array}$ & 0.44 & 0.44 \\
\hline Methionine \% & 0.48 & 0.48 \\
\hline Methionine + Cysteine \% & 0.82 & 0.82 \\
\hline Lysine \% & 1.04 & 1.04 \\
\hline
\end{tabular}

${ }^{\mathrm{a}}$ Nutrablend premix is from Premix NB 3000 used at a rate of five lbs/ton Nutra Blend LLC (Neosho,MO) consisted of manganese (4\%); zinc (4\%); iron (2\%); copper, 4.500ppm; iodine, 600ppm; selenium, 60ppm; vitamine A, 1.4E10e5 IU/lb; vitamin D3, 5.E5 IU/lb; vitamin E 3.E3 IU/lb; vitamin B12, $2 \mathrm{mg} / \mathrm{lb}$; menadione, $150 \mathrm{mg} / \mathrm{dl}$; riboflavin, $1200 \mathrm{mg} / \mathrm{dl}$; thiamine, $200 \mathrm{mg} / \mathrm{dl}$; D-pantothenic acid, $1200 \mathrm{mg} / \mathrm{dl}$; niacin; $5000 \mathrm{mg} / \mathrm{dl}$; vitamin B6 250mg/dl; folic acid 125mg/dl; choline, $700000 \mathrm{mg} / \mathrm{dl}$; Biotin $6 \mathrm{mg} / \mathrm{dl}$.

${ }^{\mathrm{b}}$ Metabolisable energy 
Table 2. Oligonucleotide primers for real-time RT-PCR

\begin{tabular}{lllcc}
\hline Gene & Forward $\left(5^{\prime}-3^{\prime}\right)$ & Reverse $\left(5^{\prime}-3^{\prime}\right)$ & Annealing $\left({ }^{\circ} \mathrm{C}\right)$ & Accession Number \\
\hline GAPDH & gagggtagtgaaggctgctg & cggatcaaaggtggaagaat & 60 & NM_204305.1 \\
GPX1 & ggaggctctgcagtaaagga & cttcgaggctttggaagatg & 60 & NM_001277853.2 \\
GPX3 & gagatcctcctgcactgaa & cacatcccctttctggaaga & 60 & NM_001163232.2 \\
$($ Cu-Zn)SOD1 & tgctggacctcgtttagctt & ctcatttcccactgccatct & 60 & NM_205064.1 \\
$(\mathrm{Mn})$ SOD2 & aatggtgggggtcatatcaa & agtcacgtttgatggcttcc & 60 & NM_204211.1 \\
$($ Cu-Zn)SOD3 & tgtgatccatgagcaggaag & gtttgccagcatttccattt & 60 & XM_015285700.1 \\
avUCP & gagaaacagagcgggatttg & ctcctggctcacggatagag & 60 & NM_204107.1 \\
\hline
\end{tabular}

${ }^{1} \mathrm{GPX}$ is Glutathione

${ }^{a} \mathrm{SOD} 2$ is mitochondrial superoxide dismutase using manganese(Mn) as an electron acceptors

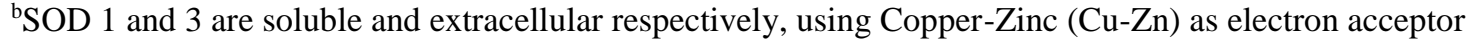

Table 3. Plasma glucose at the beginning and last week of sampling

\begin{tabular}{lcc}
\hline & Bleed 1 & Bleed 4 \\
\cline { 2 - 3 } Treatment & $\mathrm{mM}$ & $\mathrm{mM}$ \\
\hline CON & $11.5 \pm 0.45$ & $12.4 \pm 0.30$ \\
CLA & $12.9 \pm 0.45$ & $12.0 \pm 0.30$ \\
BRB & $12.0 \pm 0.45$ & $12.6 \pm 0.30$ \\
ALLO & $12.80 .45 \pm$ & $12.9 \pm 0.30$ \\
CLA+ALLO & $12.0 \pm 0.45$ & $12.7 \pm 0.30$ \\
BRB+ALLO & $12.2 \pm 0.45$ & $12.6 \pm 0.30$ \\
\hline \hline
\end{tabular}

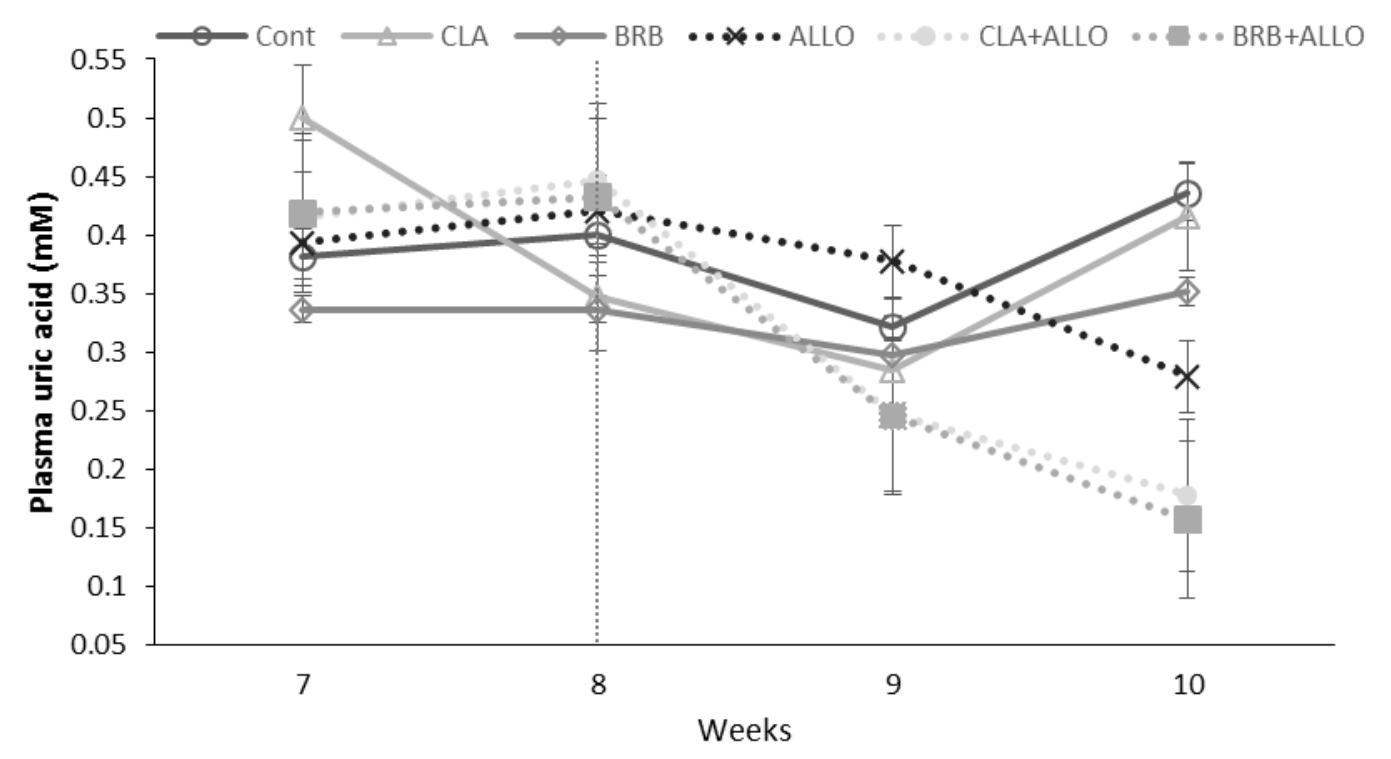


Figure 1. Change in plasma uric acid $(\mathrm{mM})$ over the course of the last four weeks of treatments
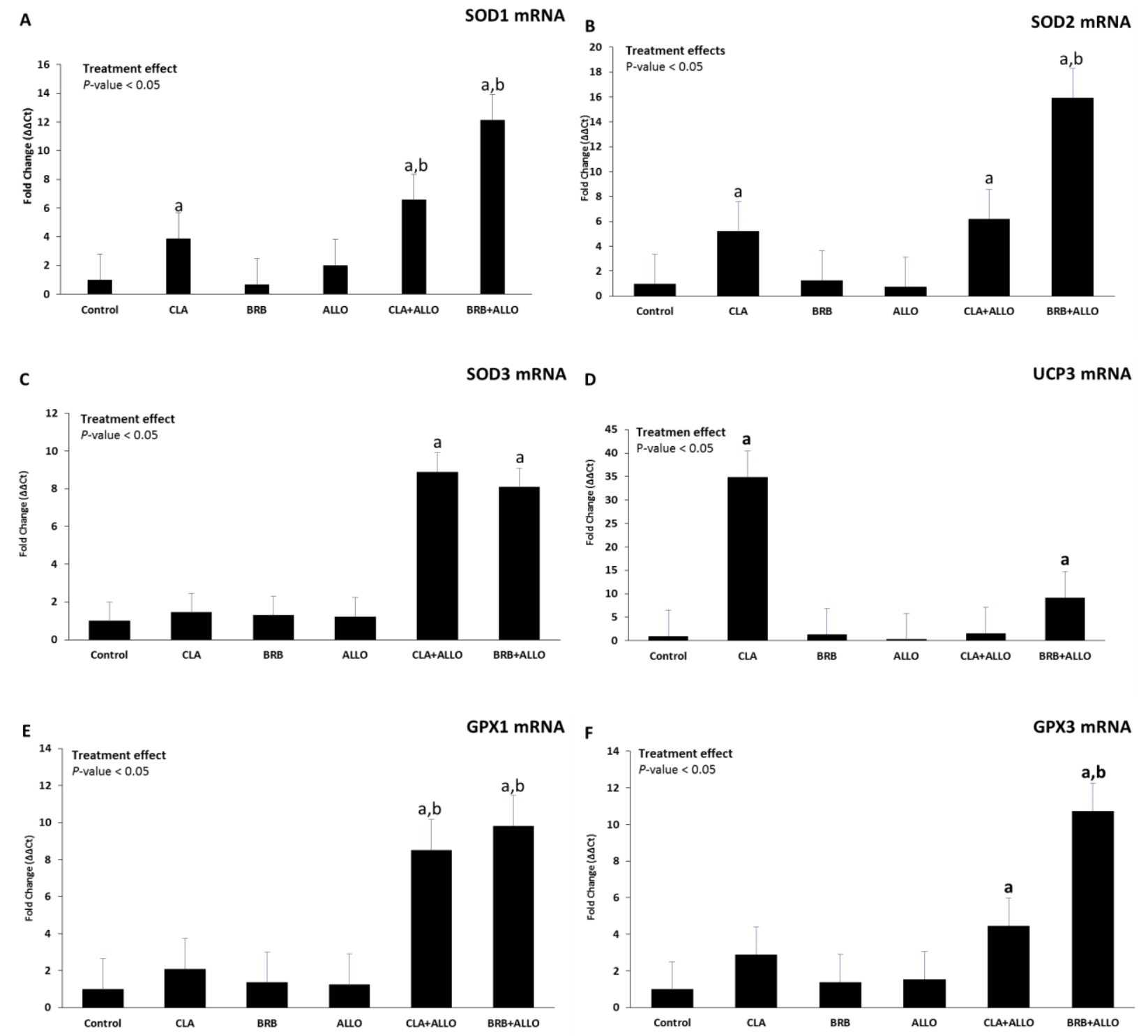

Figure 2. Relative mRNA expression of markers of oxidative stress in liver of chickens 


\section{References}

1. Federico A, Morgillo F, Tuccillo C, Ciardiello F, Loguercio C. Chronic inflammation and oxidative stress in human carcinogenesis. International Journal of Cancer. 2007 Dec 1;121(11):2381-6.

2. Cerutti P, Amstad P. Inflammation and oxidative stress in carcinogenesis. InEicosanoids and Other Bioactive Lipids in Cancer, Inflammation and Radiation Injury 1993 (pp. 387-390). Springer US.

3. Fellenberg MA, Speisky H. Antioxidants: their effects on broiler oxidative stress and its meat oxidative stability. World's Poultry Science Journal. 2006 Mar 1;62(01):53-70.

4. Chen Q, Vazquez EJ, Moghaddas S, Hoppel CL, Lesnefsky EJ. Production of Reactive Oxygen Species by Mitochondria: CENTRAL ROLE OF COMPLEX III. Journal of Biological Chemistry 2003 September 19;278(38):36027-36031.

5. Murphy MP. How mitochondria produce reactive oxygen species. Biochem J 2009 Jan 1;417(1):1-13.

6. Martin KR, Barrett JC. Reactive oxygen species as double-edged swords in cellular processes: low-dose cell signaling versus high-dose toxicity. Hum Exp Toxicol 2002 02;21(2):71-75.

7. Wu W. The signaling mechanism of ROS in tumor progression. Cancer and Metastasis Reviews 2006;25(4):- 695-705.

8. D'Autreaux B\&, Michel. ROS as signalling molecules: mechanisms that generate specificity in ROS homeostasis. Nature Reviews Molecular Cell Biology 2007;8(- 10):- 813.

9. Pisoschi AM, Pop A. The role of antioxidants in the chemistry of oxidative stress: A review. Eur J Med Chem 2015 6/5;97:55-74.

10. Simoyi MF, Falkenstein E, Van Dyke K, Blemings KP, Klandorf H. Allantoin, the oxidation product of uric acid is present in chicken and turkey plasma. Comparative Biochemistry and Physiology Part B: Biochemistry and Molecular Biology 2003;135(2):325-335.

11. Larson RA. The antioxidants of higher plants. Phytochemistry 1988;27(4):969-978.

12. Meeran SM, Katiyar S, Katiyar SK. Berberine-induced apoptosis in human prostate cancer cells is initiated by reactive oxygen species generation. Toxicol Appl Pharmacol 2008 5/15;229(1):33-43.

13. Li Z, Geng Y, Jiang J, Kong W. Antioxidant and Anti-Inflammatory Activities of Berberine in the Treatment of Diabetes Mellitus. Evidence-Based Complementary and Alternative Medicine 2014;2014:12.

14. Liu S, Lee H, Hung C, Tsai C, Li T, Tang C. Berberine attenuates CCN2-induced IL-1 $\beta$ expression and prevents cartilage degradation in a rat model of osteoarthritis. Toxicol Appl Pharmacol 2015 11/15;289(1):20-29.

15. Xie J, Xu Y, Huang X, Chen Y, Fu J, Xi M, et al. Berberine-induced apoptosis in human breast cancer cells is mediated by reactive oxygen species generation and mitochondrial-related apoptotic pathway. Tumor Biol 2015;36(2):1279-1288.

16. Teulier L, Rouanet J, Letexier D, Romestaing C, Belouze M, Rey B, et al. Cold-acclimation-induced nonshivering thermogenesis in birds is associated with upregulation of avian UCP but not with innate uncoupling or altered ATP efficiency. J Exp Biol 2010 The Company of Biologists Ltd;213(14):2476-2482 
17. Abe T, Mujahid A, Sato K, Akiba Y, Toyomizu M. Possible role of avian uncoupling protein in downregulating mitochondrial superoxide production in skeletal muscle of fasted chickens. FEBS Lett 2006 9/4;580(20):4815-4822.

18. Berardi M, Chou J. Fatty Acid Flippase Activity of UCP2 Is Essential for Its Proton Transport in Mitochondria. Cell Metabolism 2014 9/2;20(3):541-552.

19. Mollica MP, Trinchese G, Cavaliere G, De Filippo C, Cocca E, Gaita M, et al. c9,t11-Conjugated linoleic acid ameliorates steatosis by modulating mitochondrial uncoupling and Nrf2 pathway. J Lipid Res 2014 May;55(5):837-849.

20. Viladomiu M, Hontecillas R, Bassaganya-Riera J. Modulation of inflammation and immunity by dietary conjugated linoleic acid. Eur J Pharmacol 2015.

21. Yang B, Chen H, Stanton C, Ross RP, Zhang H, Chen YQ, et al. Review of the roles of conjugated linoleic acid in health and disease. Journal of Functional Foods 2015;15:314-325.

22. Ramiah SK, Meng GY, Sheau Wei T, Swee Keong Y, Ebrahimi M. Dietary Conjugated Linoleic Acid Supplementation Leads to Downregulation of PPAR Transcription in Broiler Chickens and Reduction of Adipocyte Cellularity. PPAR Res 2014;2014:137652.

23. Klandorf H, Rathore DS, Iqbal M, Shi X, Van Dyke K. Accelerated tissue aging and increased oxidative stress in broiler chickens fed allopurinol. Comparative Biochemistry and Physiology Part C: Toxicology \& Pharmacology 2001 6;129(2):93-104.

24. Settle T, Carro MD, Falkenstein E, Radke W, Klandorf H. The effects of allopurinol, uric acid, and inosine administration on xanthine oxidoreductase activity and uric acid concentrations in broilers. Poultry Science 2012 November 01;91(11):2895-2903.

25. Settle T, Falkenstein E, Klandorf H. The effect of allopurinol administration on mitochondrial respiration and gene expression of xanthine oxidoreductase, inducible nitric oxide synthase, and inflammatory cytokines in selected tissues of broiler chickens. Poultry Science 2015 October 01;94(10):2555-256.

26. Hall SB, Khudaish EA, Hart AL. Electrochemical oxidation of hydrogen peroxide at platinum electrodes. Part 1. An adsorption-controlled mechanism. Electrochim Acta 1998;43(5-6):579-588.

27. Barnes KM, Winslow NR, Shelton AG, Hlusko KC, Azain MJ. Effect of dietary conjugated linoleic acid on marbling and intramuscular adipocytes in pork. Journal of animal science. 2012 Apr 1;90(4):1142-9.

28. Van Dyke K, Castranova V. Cellular chemiluminescence. CRC; 1987 Jul 1.

29. Kong LD, Yang C, Ge F, Wang HD, Guo YS. A Chinese herbal medicine Ermiao wan reduces serum uric acid level and inhibits liver xanthine dehydrogenase and xanthine oxidase in mice. Journal of ethnopharmacology. 2004 Aug 31;93(2):325-30.

30. Takahashi Y, Kushiro M, Shinohara K, Ide T. Dietary conjugated linoleic acid reduces body fat mass and affects gene expression of proteins regulating energy metabolism in mice. Comparative Biochemistry and Physiology Part B: Biochemistry and Molecular Biology 2002;133(3):395-404. 
31. Xing L, Zhang L, Liu T, Hua Y, Zheng P, Ji G. Berberine reducing insulin resistance by up-regulating IRS2 mRNA expression in nonalcoholic fatty liver disease (NAFLD) rat liver. Eur J Pharmacol 2011;668(3):467471.

32. Cantwell H, Devery R, O'shea M, Stanton C. The effect of conjugated linoleic acid on the antioxidant enzyme defense system in rat hepatocytes. Lipids. 1999 Aug 1;34(8):833-9.

33. Halliwell B. Antioxidants in human health and disease. Annu Rev Nutr 1996;16(1):33-50.

34. Greenhalgh SN, Thompson AI, Henderson NC, Iredale JP. Oxidative Stress and Liver Inflammation. Studies on Hepatic Disorders: Springer; 2015. p. 123-147.

35. Hwang J, Wang C, Chou F, Tseng T, Hsieh Y, Lin W, et al. Inhibitory effect of berberine on tert-butyl hydroperoxide-induced oxidative damage in rat liver. Arch Toxicol 2002;76(11):664-670. 


\title{
Chapter 2
}

\begin{abstract}
Ceramides have been linked with oxidative stress in humans and dairy cows. In the poultry industry, changes in ceramide overtime and under oxidative stress state if not well known. Two compounds, conjugated linoleic acid (CLA) and berberine (BRB) a purified compound from plant root extract have been suggested to ameliorate antioxidant activity. A ten week study examined the effect of CLA and berberine supplementation on markers of oxidative stress in poultry. Broiler chickens ( $n=60)$ were equally divided into six groups; a control, a CLA group where half of the regular oil used in a standard was substituted for a CLA oil mixture of cis-9 trans-11 and trans$10 \mathrm{cis}$ - 12 isomers, a berberine group (BRB) consisting of berberine supplementation (200mg/Kg feed), an allopurinol (ALLO) group $(25 \mathrm{mg} / \mathrm{kg}$ body weight), a CLA and allopurinol (CLA+ALLO) with the same dose as the CLA and allopurinol groups and a berberine and allopurinol group (BRB+ALLO) ( $200 \mathrm{mg} / \mathrm{Kg}$ feed and $25 \mathrm{mg} / \mathrm{kg}$ body weight, respectively). The allopurinol was added to help induced an oxidative stress state. Body weight, plasma uric acid, plasma ceramide C16:0, C18:0, C20:0, C22:0 C24:0, saturated and total ceramides were measured in a time dependent manner in control and treatment effect of week 6 using GC/MS. The addition of allopurinol (ALLO) to the diet induced an oxidative stress state as measured by a significant reduction in plasma uric acid. There was a negative correlation $(r=-0.60)$ between the increase in body weight and the decrease in plasma ceramides (C16:0, C18:0 and C20:0) concentrations in control chicks. There was a decrease $(\mathrm{P}<0.05)$ in plasma ceramides $(\mathrm{C} 20: 0, \mathrm{C} 22: 0$ and $\mathrm{C} 24: 0)$ in chicks supplemented with allopurinol when compared controls. The addition of allopurinol as a mean to induce oxidative stress unwittingly affected the liver, which potentially affected the results of plasma ceramide in the oxidative stress state.
\end{abstract}


Key words: allopurinol, antioxidants, broiler, ceramide, oxidative stress,

\section{Introduction}

Compared to mammals, birds have a higher metabolism, blood glucose concentrations, body temperature as well as doubled folded mitochondria. They also have lower concentrations of insulin compared mammals (Witthow et al., 1999 ). Despite an increase in potency of chicken insulin compared to porcine (Simon et al., 1974), studies in birds have also revealed that the concentration of insulin receptors varies from tissue to tissue with muscle expressing the lowest concentration (Simon et al., 1977). These observations suggest that birds are in a constant state of insulin resistance (Simon et al., 1978). Birds, however, do not exhibit the classic symptoms associated with hyperglycemia observed in mammals, due to their elevated plasma uric acid concentrations, a potent antioxidant (Simoyi et al., 2002). Previous studies in poultry have established that allopurinol (an antagonist of xanthine and hypoxanthine in the purine degradation pathway) administration induced a redox state with an increase in inflammation associated with an increase in ROS (Simoyi et al., 2002) and proinflammatory factors (Settle et al., 2015). The severity of the redox state can be potentially adjusted by increasing dosages of allopurinol administrated (Simoyi et al., 2002). This supplementation of allopurinol in broiler chickens induces an "inflammatory state" without a "specific disease" which effectively creates a model in which to study diseases in which inflammation is predominant (e.g. Diabetes, atherosclerosis).

Ceramides (Cer) are sphingolipids composed of a sphingosine back bone attached to a fatty acid, and have long been thought of as inert component waxy lipids (Wertz et al., 1983). The acyl moiety attached to the sphingosine back bone can vary in length (16 to 24 carbons), the fatty acid 
attached is mostly dictated by the ceramide synthase homologues (1-6) involved (Bir). However, in recent years, it was discovered that they exert a variety of metabolic effects. Amongst these metabolic effects, ceramides represent a reliable biomarker of oxidative stress in mammals (Andrieu-Abadie et al., 2001, and Cutler et al., 2002). In humans, Cutler et al. (2002) linked the death of motor-neuron in amyotrophic lateral sclerosis (ALS) with an increase in ceramide accumulation in the membrane wall, which renders the cell increasingly vulnerable to oxidative stress. In dairy cows, Rico et al. (2015) established a relationship between elevated plasma ceramides (Cer C20:0 and C24:0) and prolonged insulin resistance in peripartal dairy cows. If left unchecked, the ceramides down regulated protein kinase B (Akt), a second messenger in the insulin pathway, which placed the cow at increased risk of developing metabolic diseases (Summers et al., 1998 and Rico et al., 2015). The measurement of ceramides as an early biomarker of oxidative stress could thus be beneficial to the poultry industry.

As a potential marker of oxidative stress, we measured the changes in plasma ceramides after allopurinol administration as well as the ability of two compounds, conjugated linoleic acid (CLA) and berberine (BRB), to potentially increase antioxidant (Aox) activity/expression in response the induced inflammatory state. Conjugate linoleic acid, a family of 18 carbon unsaturated fatty acids, is involved in the neutralization of ROS via the uncoupling of the oxidative phosphorylation complex in the ETC by uncoupling protein (UCP) (Echtay et al. 2001, Teulier et al. 2001). Uncoupling protein is a transmembrane protein located in the inner mitochondrial membrane. Studies of UCP have provided a mechanism by which unsaturated fatty acids (C14 to C18) enhance their activity and expression. Overall, UCP raises the proton gradient in the inner membranes, which increases the probability of any unpaired electrons to react with a proton, which prevents the formation of oxygen radicals (Diano et al. 2012, Berardi et al. 2014). The second 
compound, berberine, is an alkaloid compound isolated from plant root extract with exogenous Aox activity. This compound possesses several benzene rings, which allows the electron to circulate within the conjugated double bonds. In addition to being an exogenous AOx, BRB has been proposed to increase endogenous AOx expression and exert anti-inflammatory properties ( $\mathrm{Li}$ et al., 2014).

In view of the rapid growth rate of broilers, we hypothesized that (1) ceramide concentration should increase in broiler chickens during a production cycle (2) and that the addition of allopurinol should further should increase this production. To our knowledge, this the first time that specific species of plasma ceramides have been measured in birds. (3) Compounds which exhibit antioxidants properties, such as conjugated linoleic acid and berberine, are hypothesized to reduce the redox state induced by allopurinol and therefore decrease or lower the concentration of ceramides as compared to the allopurinol supplemented group (negative control).

\section{Materials and Methods}

All procedures and experiments were approved by West Virginia University's Institution Animal Care and Use Committee (ACUC \#15-0301.1).

\section{Experimental Design}

Sixty mixed sex day old Cobb/Cobb 500 broiler chicks were obtained from Pilgrim's Pride, Moorefield, WV. Chicks were housed within pens and maintained under a twelve hour light-dark cycle with temperature set at $27^{\circ} \mathrm{C}$. One week after hatch, chicks were divided into six groups and tagged with a leg band. The chicks were maintained on a starter diet for the first four weeks. At 
week five of the study, the broiler chicks were started on their respective treatments (see Table 1 , in Dartigue et al (submitted)): a control group, a CLA group in which half of the regular oil used in the normal diet was substituted for a mixture of 50/50 cis-9 trans-11 and trans-10 cis-12 isomers CLA oil (1.1\% CLA oil diet) (Ramiah et al., 2014). Group 3 (BRB) was supplemented with berberine $(200 \mathrm{mg} / \mathrm{kg}$ of feed) ( $\mathrm{Li}$ et al 2014), group 4 (ALLO) the diet was supplemented with allopurinol (25mg/kg of body weight) (Klandorf et al., 2001), group 5 (CLA+ALLO) and group 6 $(\mathrm{BRB}+\mathrm{ALLO})$ mimicked the CLA and BRB treatments with the addition of allopurinol $(25 \mathrm{mg} / \mathrm{kg}$ of body weight) for the last two weeks of the study. The negative control (ALLO), CLA+ALLO and BRB+ALLO were supplemented with allopurinol to help induced a state of oxidative stress (Simoyi et al., 2001, and Settle et al., 2012). Berberine (chloride hydrate, purity > 99\%) and allopurinol were purchased from Sigma-Aldrich (Saint Louis, MO, USA). The CLA was purchased commercially (Vitamorph Labs, Highland, IN, USA) as $1200 \mathrm{mg}$ capsules. Food and water were provided ad libitum.

Sampling procedure

Blood samples were obtained from the brachial wing vein of six birds per pen at week three of treatment. Each sample was placed in a heparinized tube and stored on ice. The blood samples were centrifuged at $2000 \mathrm{rpm}$ for 20 minutes at $4^{\circ} \mathrm{C}$. Plasma sample was then transferred to microcentrifuge tube and stored in a $-80^{\circ} \mathrm{C}$ freezer pending analysis.

Sample analysis 
Plasma free fatty acids concentration was measured using an enzymatic colorimetric methods (HR Series NEFA-HR, Wako chemicals USA Inc., Richmond VA). Plasma uric acid (PUA) was measured using Infinity ${ }^{\mathrm{TM}}$ Uric Acid Liquid Stable Reagent assay from Thermo Scientific (Waltham, MA, USA; Klandorf et al., 2001). Ceramide were extracted using a modified Bligh and Dyer with a C12:0 Ceramide internal standard (Avanti Polar Lipids, Alabaster, AL). Extraction and measurement of ceramide were as described in Rico et al. (2015). Concentrations of plasma ceramides C16, 18, 20, 22 and 24 were measured.

Statistical Analysis

The statistical program SAS (version 9.3; SAS Institute Inc., Cary, NC) was used to analyze the data. Both time and treatment effects were analyzed. The time-dependent data were studied as repeated measures and run as a mixed model. Weeks were considered as a fixed effect whereas birds were treated as random effect. A generalized linear model was used to study the treatment effect at week 10 of the study. The method of Kenward-Roger (1997) was used to calculate the degrees of freedom for the denominator. A measure of dependence in both time and treatment effects was performed using a Pearson's contingency coefficient. Values are expressed as least square (LSM) \pm standard error (SE) unless stated otherwise. Statistical significance was set at $P<0.05$ and 0.01 .

\section{Results}

There was a significant increase in body weight in controls (BW) over the last four weeks of the study (Figure 1, A). Plasma uric acid concentrations in controls remained constant around 
$0.4 \mathrm{mM}$, although a slight but significant $(\mathrm{P}=0.055)$ increase was noted between weeks 5 and 6 of the study (Figure 1, B). In controls, non-esterified free fatty acids stayed leveled around $200 \mu \mathrm{M}$ during weeks 3 to 5 of the study before significantly ( $\mathrm{P}<0.05$ ) falling to $100 \mu \mathrm{M}$ (Figure 1 . C).

Plasma ceramides C22 and C24 were observed to have the highest concentrations at 900 and $1000 \mathrm{ng} / \mathrm{ml}$, respectively (Figure 1, D). These were followed by plasma ceramide C16 with a concentration around $200 \mathrm{ng} / \mathrm{ml}$ (Figure 1, E). Plasma ceramides C18 and C20 were the least expressed with concentrations ranging from 30 to $70 \mathrm{ng} / \mathrm{ml}$ (Figure 1, F). The different species of plasma ceramide followed the same decreasing trend throughout the 4 weeks (Figure 1). Plasma Cer C16, C18, C20, C22, C24, as well as total saturated and total ceramides decreased $(\mathrm{P}<0.05)$ between weeks 3 and 5. However, at week 4 Ceramides C20, C22, C24, total saturated and total ceramides had significantly increased compared too weeks 3 to 5, restoring approximately $60 \%$ of the original values (Figure 1, D, F and $\mathbf{G}$ ). There were no significant changes was observed in ceramide C16 and C18 between weeks 5 and 6 (Figure 1, E and F). Notably, Cer C16, C18 and C20 concentrations were negatively correlated $(r=-0.57)$ with the increase in BW.

There were no treatment effects on BW (Figure 2, A), which was comparable to plasma Cer C18 concentration albeit, numerical differences were noted in the redox groups (Figure 2, B). Non-esterified free fatty acids measurements revealed a significant $(P<0.05)$ increase in concentrations in all treatments compared to controls (Figure 2, C). There was a significant decrease in the plasma C16 ALLO group while only numerical decreases were noted in the CLA+ALLO and BRB+ALLO groups compared to controls. Of note, the CLA+ALLO group was significantly higher than the ALLO group (Figure 2, D). Plasma ceramide species C20, C22, C24, saturated and total ceramides decreased significantly $(\mathrm{P}<0.05)$ in the allopurinol supplemented groups compared to controls (Figure 2, B, E and F). A positive correlation $(r=0.50)$ between 
PUA (Figure 2, G) and plasma ceramides C22, C24 and total ceramides behavior was also determined.

\section{Discussion and Conclusions}

Ceramides are sphingolipids that have been positively associated with oxidative stress and metabolic diseases in mammals (Andrieu-Abadie et al., 2001, and Cutler et al., 2002) but their role in birds has not been established. A redox state in birds can be induced by a variety of factors, amongst them allopurinol (Klandorf et al., 2001, Simoyi et al., 2002), with a shift towards prooxidants which can be detrimental. Previous research has demonstrated that allopurinol can reduce liver uric acid production by inhibiting liver xanthine oxidase (Simoyi et al., 2002, Carro et al., 2009, and Settle et al., 2012). The decrease in PUA, a potent antioxidants in birds, can lead to state of oxidative stress characterized by overall inflammation but also liver inflammation (Carro et al., 2009, Settle et al., 2015). In addition, Carro et al. (2009) determined that various doses of allopurinol could change the severity of the redox state, effectively creating a somewhat controllable model of oxidative stress. We made use of this model to investigate plasma ceramides in broiler chickens. Changes in plasma ceramide concentrations were first determined during the growth phase and in response to different treatments.

Measurement of plasma ceramides in control birds over the course of 4 weeks revealed an overall significant decline in plasma ceramides although between week 5 and 6 some species significantly increased (C22:0 and C24:0) whereas others (C16:0, C18:0 and C20:0) remained depressed. These results did not support our initial hypothesis, as plasma ceramide concentrations increased with age in broiler chicks as has been observed in other species (Lightle et al., 2000). 
These observations may result from a decrease in the growth rate/metabolic rate observed at 5 weeks of age in broiler chickens as has been previously described (Kuenzel et al., 1977). Schols et al. (1996) suggested that an increase in basal metabolic rate was associated with an increase in the inflammatory state in humans as indicated by the increase in inflammatory markers. Hence, the downturn in metabolic rate and decrease in oxygen $\left(\mathrm{O}_{2}\right)$ consumption is suggested to parallel the decrease in plasma ceramides concentrations. If the downtrend was prolonged past week 5 of age as measured by Kuenzel et al. (1977) this would support the negative correlation $(r=-0.57)$ observed between the increase in body weight and the decrease in plasma ceramide concentrations.

The liver is primary organ for free fatty acid synthesis in birds (Whittow et al., 1999). Ceramide synthesis occurs via the addition of a fatty acid moiety to a sphingosine back bone via ceramide synthase (Bikman et al., 2011). In this study, non-esterified fatty acids concentrations were relatively constant until a significant decline $(\mathrm{P}<0.05)$ was observed between weeks 5 and 6 , with this decrease reciprocally paralleling the increase in plasma ceramides during this same period. Watt et al. (2012) demonstrated that ceramide synthesis was dependent on substrate availability rather than liver serine palmitoyltransferase (SPT) activity. These results suggest that a fraction of avalaible free fatty acids may have been shuttled towards ceramide synthesis, while free fatty acids levels were not restored to their original levels. As ceramides are markers of oxidative stress and positively correlated with inflammation characterized by ROS production (Andrieu-Abadie et al., 2001, Villena et al., 2007), this scenario could also account for the increase $(\mathrm{P}<0.055)$ in PUA, a potent antioxidant, during that period.

The induction of an inflammatory state by allopurinol was evident by the significant decreases in plasma uric acid. However, (2) plasma ceramides in the allopurinol fed groups did not increase as predicted, rather there was a significant decrease in most species measured. The 
inflammatory state is associated with an increase in protein synthesis as observed in rats, including various inflammatory proteins such as interleukins and their counterparts (anti-inflammatory and antioxidants proteins) (Birch et al., 1986, Vary et al., 1992, Knight et al., 2005) . This increase in liver protein synthesis coupled with the decrease in feed intake caused by allopurinol may have contributed to the decrease in plasma ceramides concentrations. Comparably, serine amino acids, which are required for ceramide synthesis, could be redirected towards protein synthesis as well (Birch et al., 1986, Vary et al., 1992). Schreiber et al. (1982) demonstrated that a labeled pool of liver leucine in rats was incorporated into plasma proteins, for up to 3 days after the challenge as result of inducing inflammation.

NEFA were significantly elevated in all treatments compared to controls. This increase in NEFA may be a result of the inclusion of CLA, BRB and allopurinol in the diet. BRB has been suggested to inhibit adipogenesis in mammals, (Choi et al., 2006, Hu et al., 2010) which could explain the observed increase in NEFA. Conjugated linoleic acid is known to increase the rate of lipolysisin adipose tissues, which would explain the increase in circulating fatty acids (REFERENCE). Although similar results were observed in Moulard ducks (hybrid of Muscovy and Pekin ducks) (Felser et al., 2013); a decrease in total hepatic NEFA (which would suggest a decrease in serum NEFA) was observed in chickens fed CLA (Ko et al., 2004). Previous studies in ApoE KO mice supplemented with $10 \mu \mathrm{M}$ of allopurinol for 4 weeks revealed a numerical increase in serum NEFA, which was suggested to be the result of allopurinol inhibiting the uptake of low density lipoprotein (LDL) into macrophages. In a second study the authors increased the expression of xanthine oxidoreductase in cell cultures which increased LDL uptake in macrophage cells resulting in foam cell formation (Kushiyama et al., 2012). The combined synergistic effect of BRB+ALLO would explain the difference $(P=0.0504)$ in NEFA observed between the BRB 
and BRB+ALLO groups. However, only a numerical increase in NEFA was noted in the CLA+ALLO group compared to CLA.

Both plasma ceramide C16 and C18 in controls and treatments were the least changed compared to the other ceramides species. There was a significant linear decrease in C16 and C18 in controls. However, there was a numerical decrease in C16 and C18 concentrations when challenged with allopurinol. Ceramides $\mathrm{C} 16$ and $\mathrm{C} 18$ have been linked to insulin resistance. Turpin et al 2014 demonstrated that an increase in Cer C16 further aggravated the insulin resistance state in mice. In a study where Ceramide synthase 6 (CerS6) knockout mice were used to investigate the absence of Cer $\mathrm{C} 16$, the authors observed a decrease in the ceramide C16 coupled with an improvement in insulin sensitivity and an increase energy expenditure. If Cer C16:0's metabolic effects were comparable to avian species, the concentrations measured in the present study might help to explain why birds are in a constant state of hyperglycemia. In addition, Ceramide synthase (CerS) 6 and 5 (producers of Cer C16:0 and C18:0) are equally expressed in a greater number of tissues than Cers 2 and 4 (producers of Cer C20:0- C24:0) which are mostly expressed in liver and kidneys (Grösch et al., 2011). If this pattern of expression is similar in birds, this might explain the decrease in plasma ceramides C20 to C24 in the broiler chickens fed allopurinol.

Measurement of ceramide in broiler chickens as markers of oxidative stress is feasible as they have already been correlated with oxidative stress and ROS production in other species. In addition, treatments for some ceramide specific associated diseases have already been devised. In human patients with amyotrophic lateral sclerosis (ALS), the authors observed an increase and accumulation of Cer C16 in neurons which resulted in the death of the neurons. They also demonstrated that treatment with the antibiotic myroicin, an inhibitor of SPT, resulted in a decrease in Cer 16 accumulation in cells (Culter et al., 2002). A provisional analysis of ceramides in 
growing broiler chickens, revealed a deecline towards the end of the growth phase (weeks 2 to $\sim 8$ of age) (Kuenzel et al., 1977). Under conditions of oxidative stress, most species of ceramides are decreased, however, this may be an indirect consequence of inducing the inflammatory state. The use of allopurinol although practical specifically targets the liver which in birds, is the primary organ for fatty acids synthesis. For this reason, ceramide production might have been unwittingly affected by the addition of allopurinol. Alternative methods to induce an oxidative stress state challenge should be investigated to corroborate the findings of this study. 
Figures

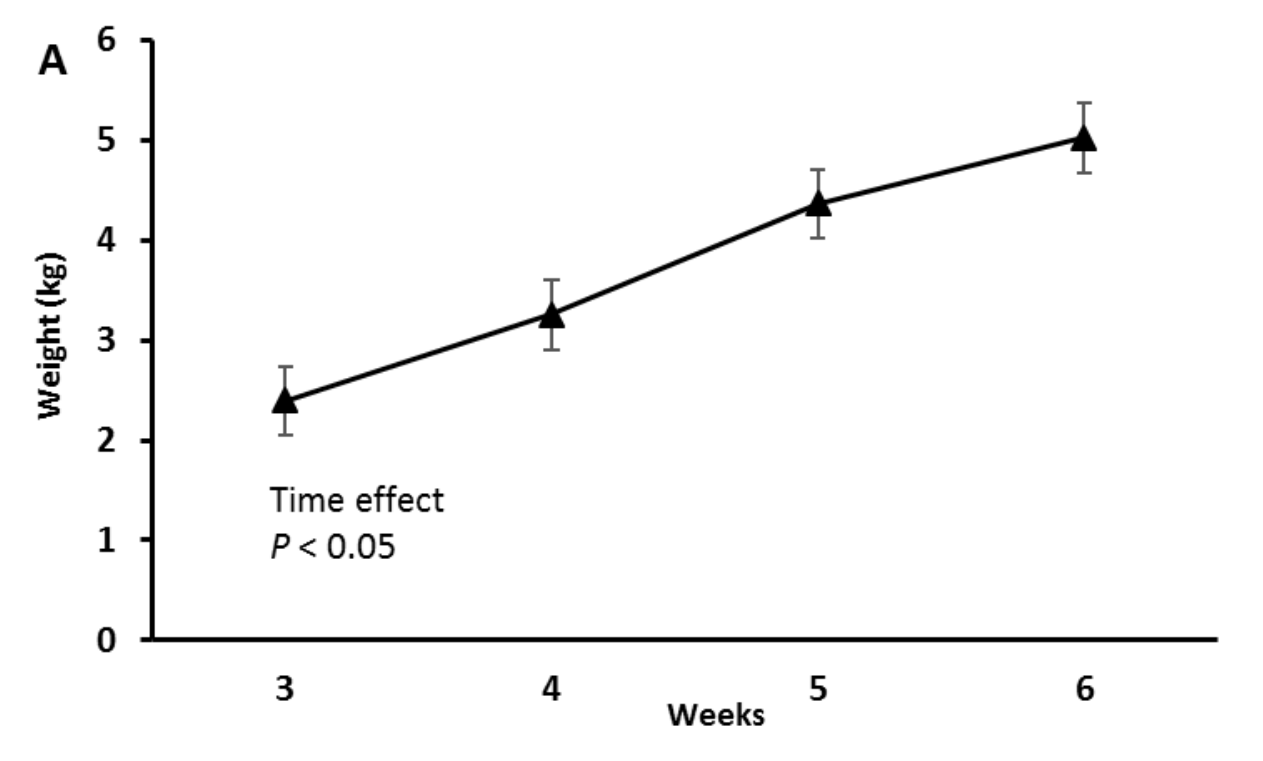

B

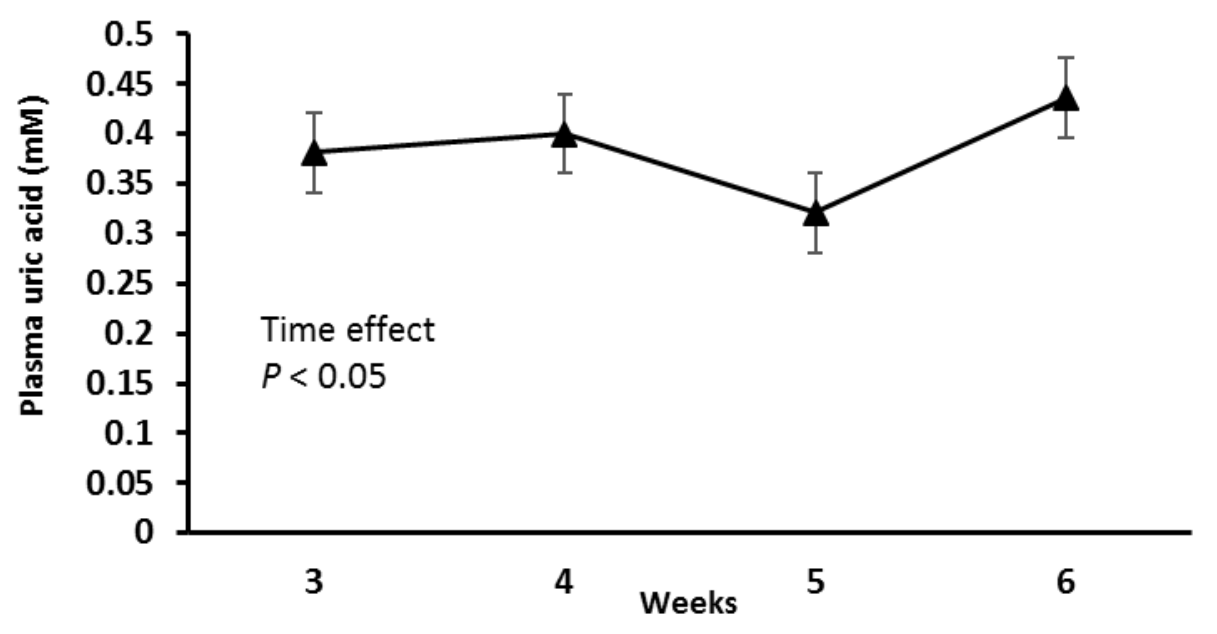



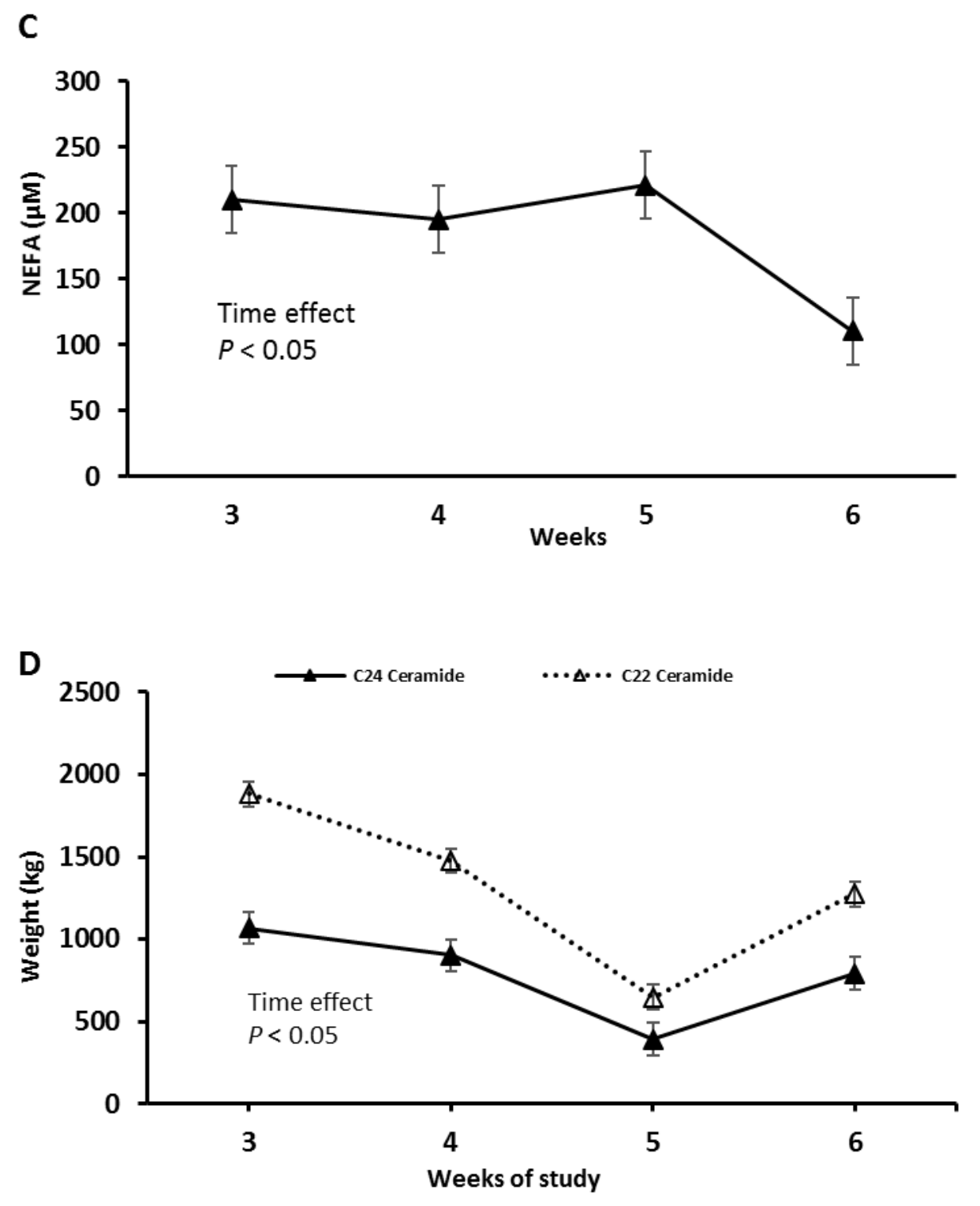

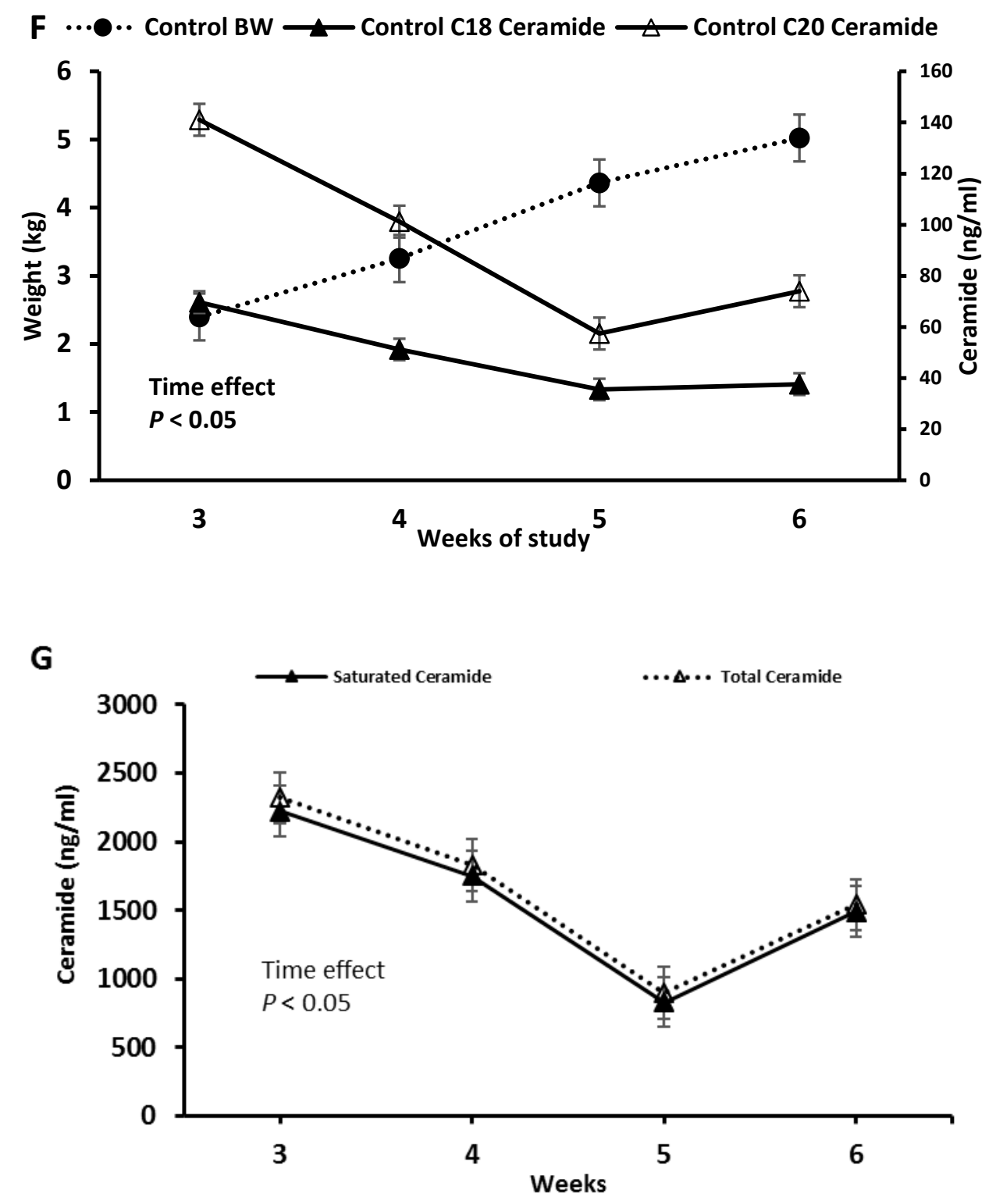


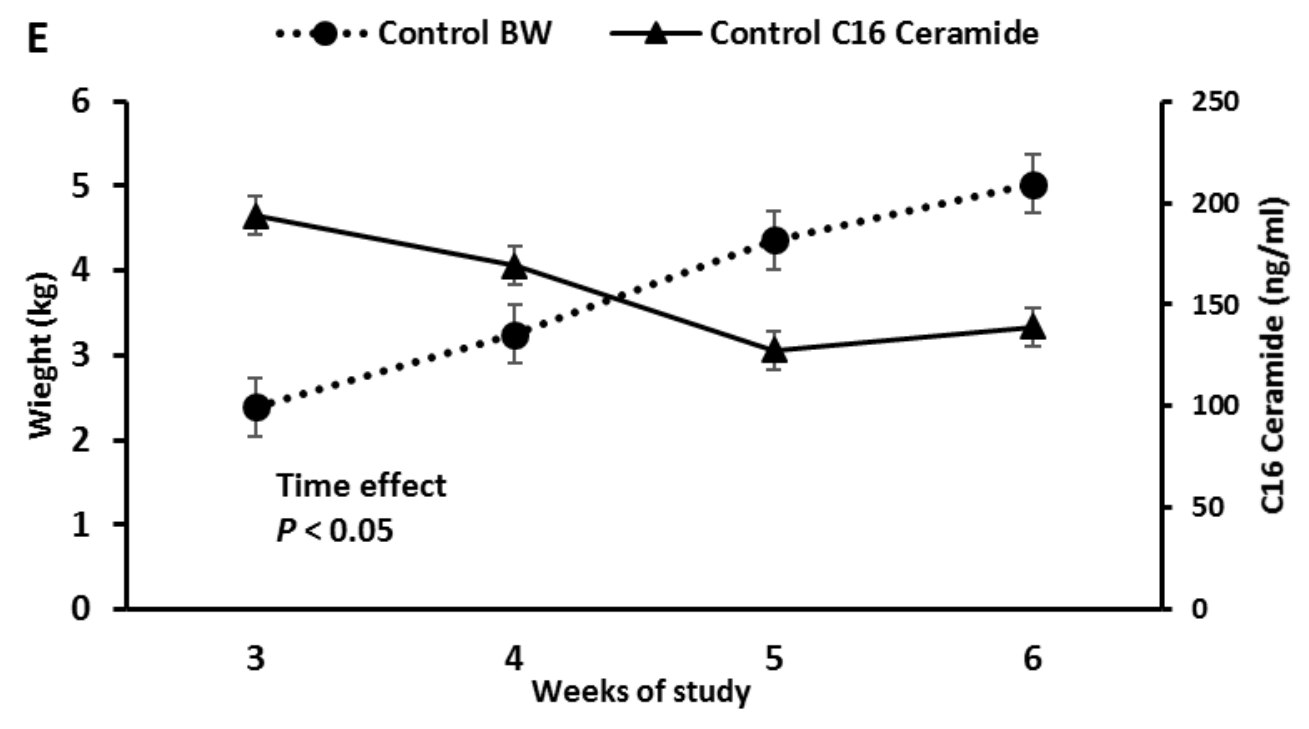

Figure 1. Changes in plasma ceramides, PUA, NEFA and weight in control birds over time

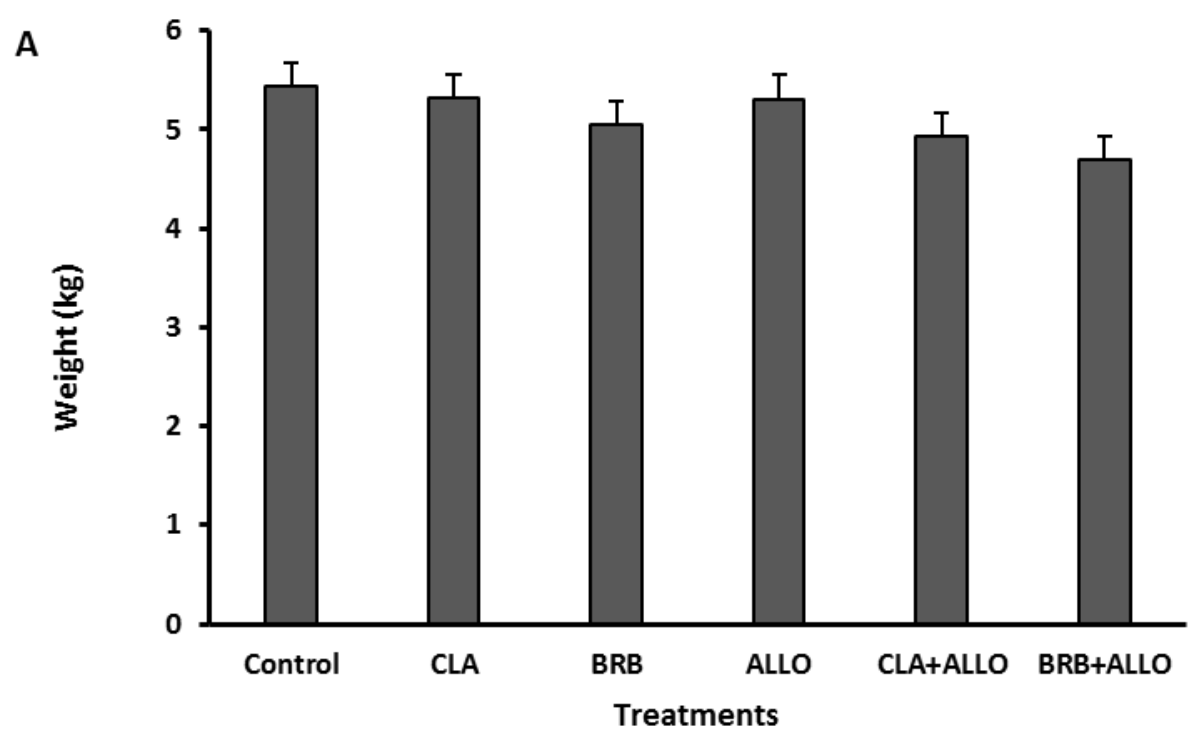



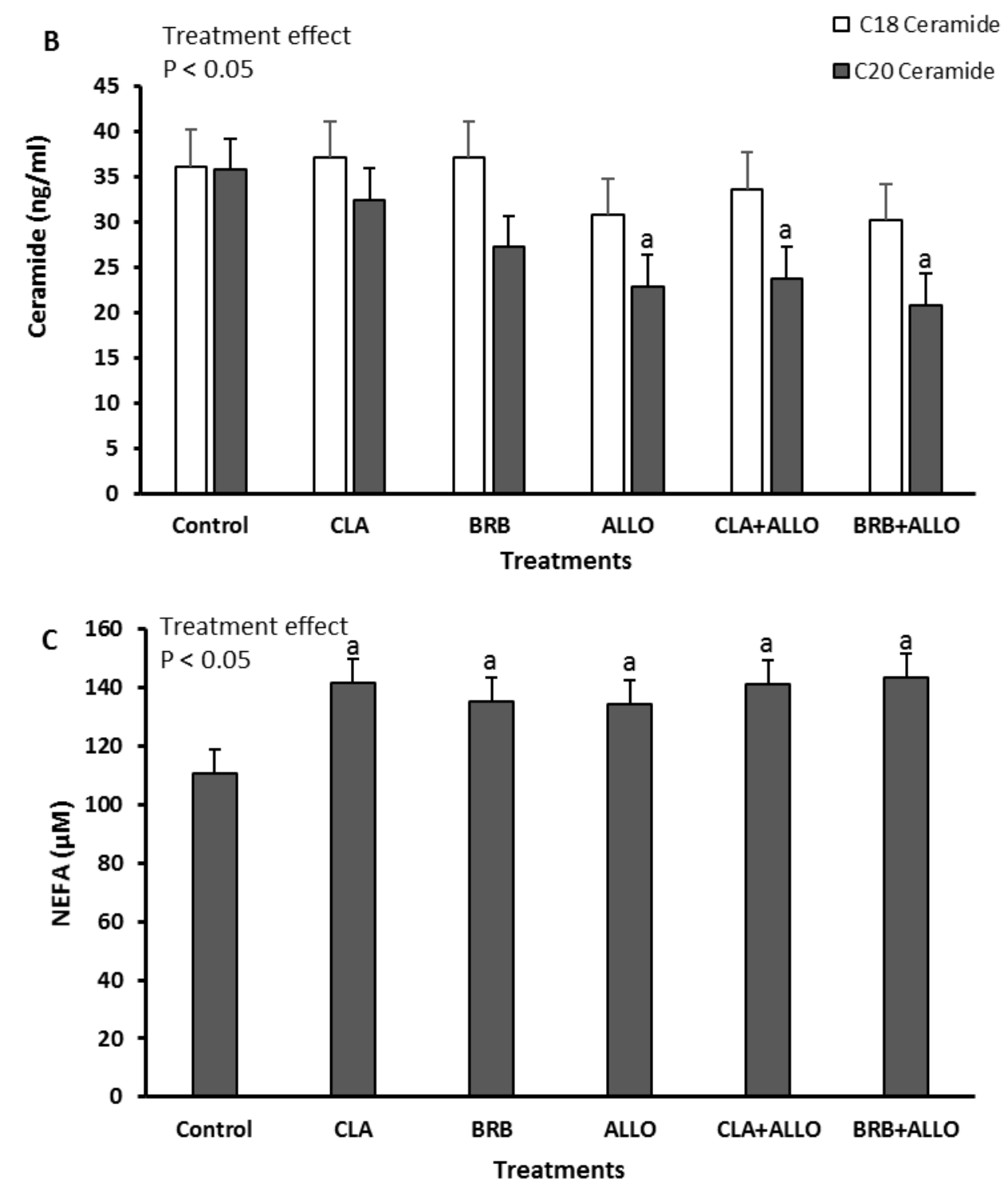

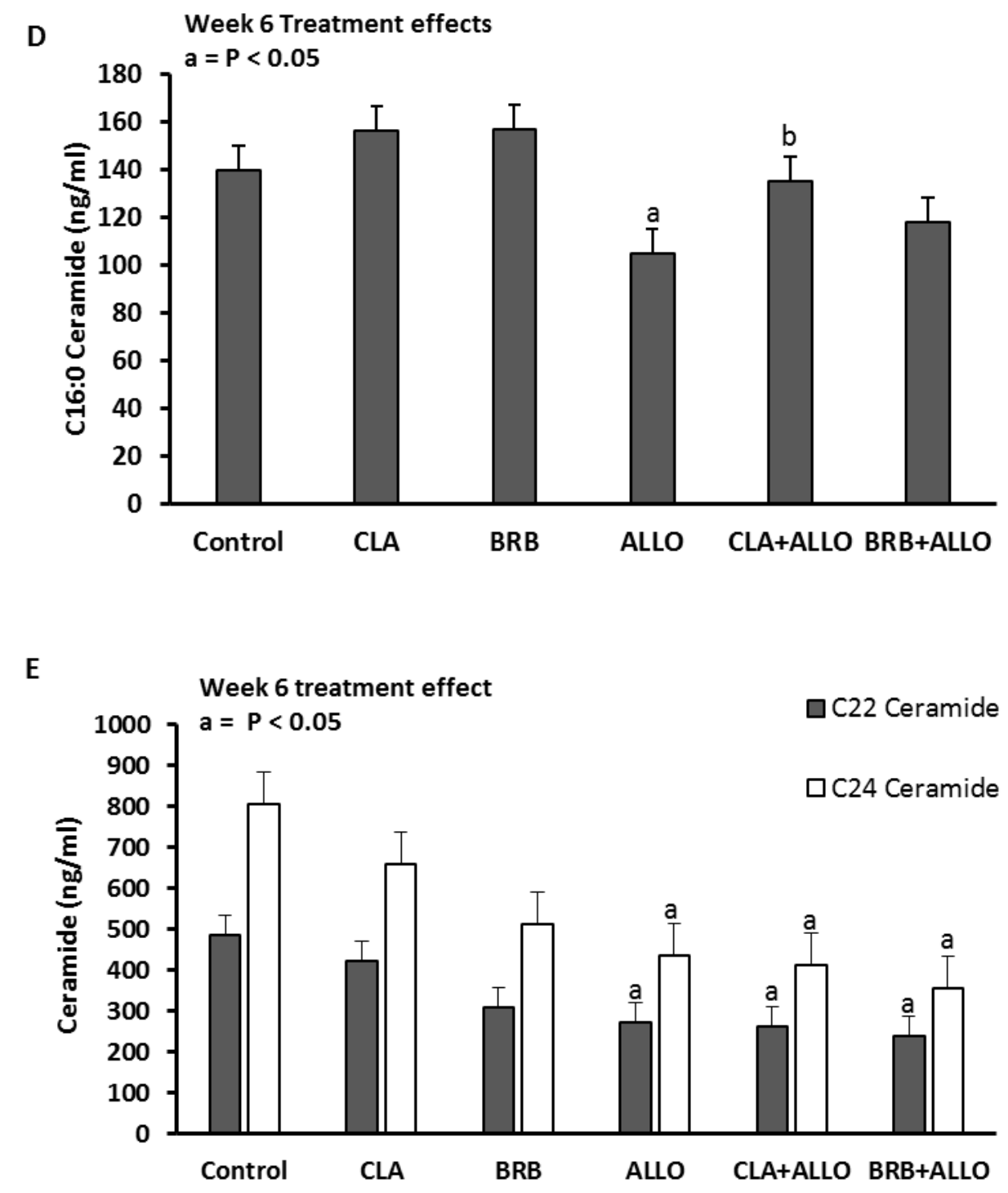

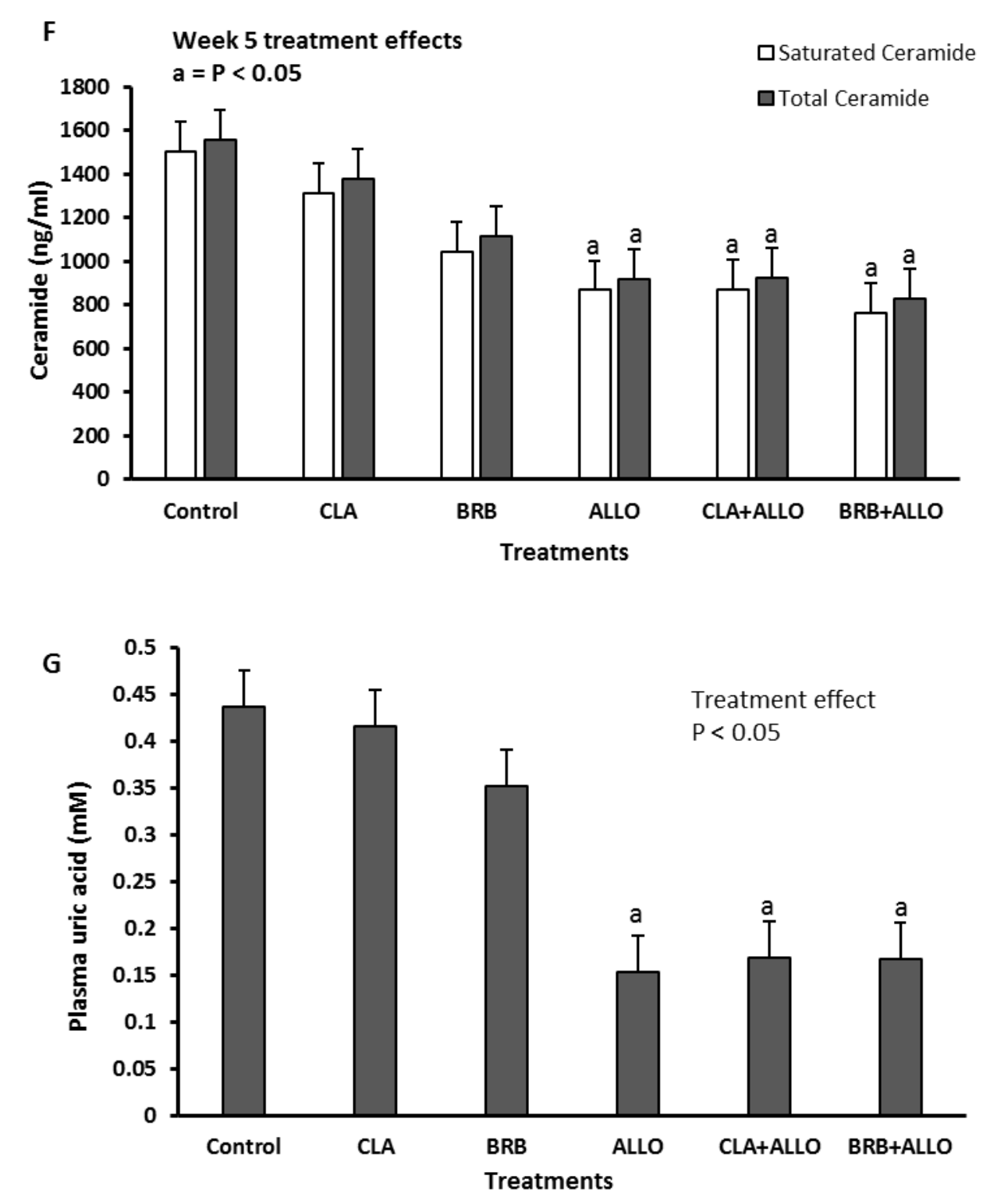

Figure 2. Treatment effects on body weight, plasma ceramides, PUA and NEFA

Figure 1. Plasma concentrations of $(\mathbf{A}, \mathbf{B}, \mathbf{C}$, and $\mathbf{D})$ ceramides, $(\mathbf{E})$ NEFA, and $(\mathbf{F})$ plasma uric acid in control broiler chickens over-time. Overall for panel $\mathbf{A}, \mathbf{B}, \mathbf{C}$ and $\mathbf{D}$ there was a significant decrease in plasma ceramide concentrations over the course of the first three weeks (7,8 and 9) of bleed. In $\mathbf{A}$, there was increase although not significant in $\mathrm{C} 16$ ceramide compared to the previous week. The same pattern was observed in panel $\mathbf{B}$ for C18 ceramide. In the case of $\mathrm{C} 20$ ceramide (also B), there was significant increase from the $9^{\text {th }}$ to the $10^{\text {th }}$ weeks, this pattern was also observed in C24 and C22 ceramide (C) and saturated and total ceramides (D). There was decrease in NEFA (E) with a significant decrease observed from week 9 to 10 . For plasma uric acid, the concentration were pretty similar until week 9; between week 9 and 10, there was significant increase. $P<0.05$. 
Figure 2. Body weight (A), plasma ceramide concentrations of (B,D,E,F) ceramide, $(\mathbf{G})$ uric acid and (B) NEFA, of broiler chicken on week 10 of treatment. Panel A represent the body weight, there were no significant changes to report. (B) There were no significicant differences in plasma ceramide 18, in constrast all the groups supplemented with ceramide C20:0 were significantly decrease compared to controls. (C)The measurement of NEFA revealed a significant increase in all groups compared to control In panel $\mathbf{D}$, there was a significant decrease in C16 ceramide in the allopurinol group compared to control and a significant difference between ALLO and the CLA+ALLO group. In addition, a numerical decrease in the CLA+ALLO and BRB+ALLO group was noted. Panel $\mathbf{E}$ and $\mathbf{F}$ followed the same pattern, there was a significant decrease in plasma ceramides and plasma uric acid concentrations in the ALLO, CLA+ALLO and BRB+ALLO groups compared to control. The notation a represents significance compared to control whereas b compares to the ALLO group. $P<0.05$

\section{References}

1. Whittow GC, editor. Sturkie's avian physiology. Academic Press; 1999 Oct 14.

2. Simon J. Chicken as a useful species for the comprehension of insulin action. Critical reviews in poultry biology (USA). 1989.

3. Simon J, Rosebrough RW, McMurtry JP, Steele NC, Roth J, Adamo M, LeRoith D. Fasting and refeeding alter the insulin receptor tyrosine kinase in chicken liver but fail to affect brain insulin receptors. Journal of Biological Chemistry. 1986 Dec 25;261(36):17081-8.

4. Simoyi MF, Falkenstein E, Van Dyke K, Blemings KP, Klandorf H. Allantoin, the oxidation product of uric acid is present in chicken and turkey plasma. Comparative Biochemistry and Physiology Part B: Biochemistry and Molecular Biology 2003;135(2):325-335

5. Settle T, Falkenstein E, Klandorf $H$. The effect of allopurinol administration on mitochondrial respiration and gene expression of xanthine oxidoreductase, inducible nitric oxide synthase, and inflammatory cytokines in selected tissues of broiler chickens. Poultry Science 2015 October 01;94(10):2555-256.

6. Wertz PW, Downing DT. Ceramides of pig epidermis: structure determination. Journal of lipid research. 1983 Jun 1;24(6):759-65.

7. Cutler, R. G., Pedersen, W. A., Camandola, S., Rothstein, J. D., \& Mattson, M. P. (2002). Evidence that accumulation of ceramides and cholesterol esters mediates oxidative stress-induced death of motor neurons in amyotrophic lateral sclerosis. Annals of neurology, 52(4), 448-457.

8. Andrieu-Abadie, N., Gouazé, V., Salvayre, R., \& Levade, T. (2001). Ceramide in apoptosis signaling: relationship with oxidative stress. Free Radical Biology and Medicine, 31(6), 717-728.

9. Rico JE, Bandaru VV, Dorskind JM, Haughey NJ, McFadden JW. Plasma ceramides are elevated in overweight Holstein dairy cows experiencing greater lipolysis and insulin resistance during the transition from late pregnancy to early lactation. Journal of dairy science. 2015 Nov 30;98(11):7757-70. 
10. Summers SA, Garza LA, Zhou H, Birnbaum MJ. Regulation of insulin-stimulated glucose transporter GLUT4 translocation and Akt kinase activity by ceramide. Molecular and cellular biology. 1998 Sep 1;18(9):5457-64.

11. Echtay KS, Winkler E, Frischmuth K, Klingenberg M. Uncoupling proteins 2 and 3 are highly active $\mathrm{H}+$ transporters and highly nucleotide sensitive when activated by coenzyme $\mathrm{Q}$ (ubiquinone). Proceedings of the National Academy of Sciences 2001 February 13;98(4):14161421.

12. Teulier L, Rouanet J, Letexier D, Romestaing C, Belouze M, Rey B, et al. Cold-acclimationinduced non-shivering thermogenesis in birds is associated with upregulation of avian UCP but not with innate uncoupling or altered ATP efficiency. J Exp Biol 2010 The Company of Biologists Ltd;213(14):2476-2482.

13. Diano S, Horvath TL. Mitochondrial uncoupling protein 2 (UCP2) in glucose and lipid metabolism. Trends Mol Med 2012 1;18(1):52-58.

14. Berardi M, Chou J. Fatty Acid Flippase Activity of UCP2 Is Essential for Its Proton Transport in Mitochondria. Cell Metabolism 2014 9/2;20(3):541-552.

15. Li Z, Geng YN, Jiang JD, Kong WJ. Antioxidant and anti-inflammatory activities of berberine in the treatment of diabetes mellitus. Evid Based Complement Alternat Med 2014;2014:289264.

16. Klandorf H, Rathore DS, Iqbal M, Shi X, Van Dyke K. Accelerated tissue aging and increased oxidative stress in broiler chickens fed allopurinol. Comparative Biochemistry and Physiology Part C: Toxicology \& Pharmacology 2001 6;129(2):93-104. Ramiah SK, Meng GY, Sheau Wei T, Swee Keong Y, Ebrahimi M. Dietary Conjugated Linoleic Acid Supplementation Leads to Downregulation of PPAR Transcription in Broiler Chickens and Reduction of Adipocyte Cellularity. PPAR Res 2014;2014:137652.

17. Settle T, Carro MD, Falkenstein E, Radke W, Klandorf H. The effects of allopurinol, uric acid, and inosine administration on xanthine oxidoreductase activity and uric acid concentrations in broilers. Poultry Science 2012 November 01;91(11):2895-2903.

18. Andrieu-Abadie, N., Gouazé, V., Salvayre, R., \& Levade, T. (2001). Ceramide in apoptosis signaling: relationship with oxidative stress. Free Radical Biology and Medicine, 31(6), 717-728.

19. Carro MD, Falkenstein E, Radke WJ, Klandorf H. Effects of allopurinol on uric acid concentrations, xanthine oxidoreductase activity and oxidative stress in broiler chickens. Comparative Biochemistry and Physiology Part C: Toxicology \& Pharmacology 2010 1;151(1):12-17.

20. Bikman BT, Summers SA. Ceramides as modulators of cellular and whole-body metabolism. J Clin Invest 2011 Nov;121(11):4222-4230. 
21. Lightle SA, Oakley JI, Nikolova-Karakashian MN. Activation of sphingolipid turnover and chronic generation of ceramide and sphingosine in liver during aging. Mechanisms of ageing and development. 2000 Dec 1;120(1):111-25.

22. Kuenzel WJ, Kuenzel NT. Basal metabolic rate in growing chicks Gallus domesticus. Poultry Science. 1977 Mar 1;56(2):619-27.

23. Schols AM, Buurman WA, Van den Brekel AS, Dentener MA, Wouters EF. Evidence for a relation between metabolic derangements and increased levels of inflammatory mediators in a subgroup of patients with chronic obstructive pulmonary disease. Thorax. 1996 Aug 1;51(8):819-24.

24. Watt MJ, Barnett AC, Bruce CR, Schenk S, Horowitz JF, Hoy AJ. Regulation of plasma ceramide levels with fatty acid oversupply: evidence that the liver detects and secretes de novo synthesised ceramide. Diabetologia. 2012 Oct 1;55(10):2741-6.

25. Knight B, Matthews VB, Akhurst B, Croager EJ, Klinken E, Abraham LJ, Olynyk JK, Yeoh G. Liver inflammation and cytokine production, but not acute phase protein synthesis, accompany the adult liver progenitor (oval) cell response to chronic liver injury. Immunology and cell biology. 2005 Aug 1;83(4):364-74.

26. Vary TC, Kimball SR. Regulation of hepatic protein synthesis in chronic inflammation and sepsis. American Journal of Physiology-Cell Physiology. 1992 Feb 1;262(2):C445-52.

27. Birch HE, Schreiber G. Transcriptional regulation of plasma protein synthesis during inflammation. Journal of Biological Chemistry. 1986 Jun 25;261(18):8077-80.

28. Schreiber G, Howlett G, Nagashima M, Millership A, Martin H, Urban J, Kotler L. The acute phase response of plasma protein synthesis during experimental inflammation. Journal of Biological Chemistry. 1982 Sep 10;257(17):10271-7.

29. Choi BH, Ahn IS, Kim YH, Park JW, Lee SY, Hyun CK, Do MS. Berberine reduces the expression of adipogenic enzymes and inflammatory molecules of 3T3-L1 adipocyte. Experimental \& molecular medicine. 2006 Dec 1;38(6):599.

30. $\mathrm{Hu} \mathrm{Y,} \mathrm{Davies} \mathrm{GE.} \mathrm{Berberine} \mathrm{inhibits} \mathrm{adipogenesis} \mathrm{in} \mathrm{high-fat} \mathrm{diet-induced} \mathrm{obesity} \mathrm{mice.}$ Fitoterapia. 2010 Jul 31;81(5):358-66.

31. Fesler JA, Peterson DG. Conjugated linoleic acids alter body composition differently according to physiological age in Moulard ducks. Poultry science. 2013 Oct 1;92(10):2697-704.

32. Ko YH, Yang HY, Jang IS. Effect of conjugated linoleic acid on intestinal and hepatic antioxidant enzyme activity and lipid peroxidation in broiler chickens. Asian Australasian Journal of Animal Sciences. 2004 Jan 1;17(8):1162-7.

33. Kushiyama A, Okubo H, Sakoda H, Kikuchi T, Fujishiro M, Sato H, Kushiyama S, Iwashita M, Nishimura F, Fukushima T, Nakatsu Y. Xanthine oxidoreductase is involved in macrophage foam cell formation and atherosclerosis development. Arteriosclerosis, Thrombosis, and Vascular Biology. 2012 Feb 1;32(2):291-8.

34. Grösch S, Schiffmann S, Geisslinger G. Chain length-specific properties of ceramides. Progress in lipid research. 2012 Jan 31;51(1):50-62. 